\title{
Reorganization of self-assembled DNA-based polymers using orthogonally addressable building blocks
}

Serena Gentile, ${ }^{[a]}$ Erica del Grosso, ${ }^{[a]}$ Leonard J. Prins, ${ }^{[b]}$ Francesco Ricci ${ }^{[a], *}$

[a] Department of Chemistry, University of Rome, Tor Vergata, Via della Ricerca Scientifica, 00133 Rome, Italy,

[b] Department of Chemical Sciences, University of Padua, Via Marzolo 1, 35131 Padua, Italy

*Corresponding author: francesco.ricci@uniroma2.it

\begin{abstract}
Nature uses the breaking and making of non-covalent interactions to achieve the reversible formation and structural reconfiguration of biopolymers, a strategy that permits dynamic adaptation in response to external cues and to changes in the environment. Inspired by this observation and taking advantage of the addressability and programmability of DNA/DNA non-covalent interactions we report here the rational design of orthogonal DNA-based addressable tiles that self-assemble into polymer-like structures that can be reconfigured and reorganized by external inputs. The different tiles share the same 5-nucleotide sticky ends responsible for self-assembly but are rationally designed to contain a specific regulator-binding domain that can be orthogonally targeted by different DNA regulator strands (activators and inhibitors). We show that by sequentially adding specific activators and inhibitors it is possible to re-organize in a dynamic and reversible way the formed polymer-like structures to display well-defined distributions: homopolymers made of a single tile, random polymers in which different tiles are distributed randomly and block structures in which the tiles are organized in segments. The versatility of the systems presented in this study shows the ease with which DNA-based addressable monomers can be designed to create reconfigurable synthetic micron-scale DNA structures offering a new approach to the growing field of supramolecular polymers.
\end{abstract}




\section{Introduction}

Nature employs both covalent and non-covalent interactions to achieve control over the formation and function of different biopolymers with extraordinary efficiency. ${ }^{1}$ While covalent interactions provide a means to achieve stable and durable materials, noncovalent interactions are crucial to allow structural dynamic reconfiguration, environmental adaptation and reversibility. ${ }^{2-6}$ Inspired by these sophisticated mechanisms, novel manmade materials have been recently described that increasingly make use of non-covalent interactions for the reversible and controllable modulation of their properties. ${ }^{7-11}$ In this context, a strong current interest in the field of supramolecular chemistry regards the rational design of building blocks that can self-assemble into supramolecular polymers through dynamic non-covalent interactions. ${ }^{12-17} \mathrm{~A}$ wide range of non-covalent interactions including hydrogen bonds, ${ }^{18,19}$ hydrophobic, ${ }^{20}$ stacking interactions ${ }^{21,22}$ and metal-ligand coordination, ${ }^{23}$ has been employed for this purpose. Similarly to what occurs in natural systems, the reversible non-covalent nature of these interactions leads to supramolecular polymers that can respond to environmental, chemical and biological stimuli. ${ }^{24-31}$ It also allows the reconfiguration of the polymer structure by changing the order in which the monomer units self-assemble. ${ }^{32}$ Although several examples have appeared in the literature in which different addressable monomers have been used to reconfigure supramolecular polymers using different inputs, ${ }^{32-38}$ the challenge remains to rationally design the monomers in such a way to permit a predictable and versatile reconfiguration of the polymer with a high degree of control.

Synthetic nucleic acid strands (DNA and RNA) have emerged as ideal components for self-assembly processes. The high programmability and the possibility to predict in a straightforward way the thermodynamics of the involved non-covalent hydrogen bond base pairings, together with the low cost of synthesis, has allowed the self-assembly of unprecedented precise 2D and 3D structures, hydrogels, nanodevices and polymers from rationally designed synthetic DNA oligonucleotides. ${ }^{39-45}$ Recently, the possibility to reconfigure these structures has also been demonstrated enabling dynamic DNA structures with potential adaptive behaviour. ${ }^{46-51}$

Motivated by the above arguments and taking advantage of the addressability of DNA we show here that synthetic nucleic acids are particularly suited for designing selfassembling dynamic polymer-like structures that can be easily reconfigured and reorganized by external inputs. To do this we have rationally designed monomer units that can be orthogonally addressed by different DNA regulator strands and can be used to 
structurally reorganize the polymers between homopolymers, random co-polymers and diand tri-block co-polymers (Fig. 1d). The versatility of the systems presented in this study shows the ease at with DNA-based supramolecular polymers can be controlled using external triggers. 


\section{Results and Discussion}

\section{Rational design of orthogonally addressable DNA tiles}

To demonstrate rational reconfiguration of DNA-based polymers we employed a design in which DNA tiles, formed through the interaction of five different DNA strands, self-assemble at room temperature into hollow tubular polymeric structures. ${ }^{52}$ The structures form through the non-covalent interactions between four sticky ends (each of 5 nucleotides) and can reach a length in the order of a few micrometres (Fig.1a). ${ }^{52,53}$ The capacity of these tiles to self-assemble can be controlled by the addition of regulator strands (Fig. 1b) ${ }^{54,55}$ For example, the addition of an inhibitor DNA strand (originally named invader) $)^{54,55}$ able to bind one of the four sticky ends of the tile allows to completely inactivate it so that self-assembly capacity is inhibited. ${ }^{54,55}$ Such invader strand can also lead to the disassembly of an already formed polymeric structure (Fig. 1b, S1) ${ }^{54,55}$ On the other hand, tiles can be re-activated for self-assembly by an activator DNA strand (originally named anti-invader) that, through a toehold strand displacement reaction, displaces the invader from the inactive tile (Fig.1b, S2). ${ }^{54,55}$

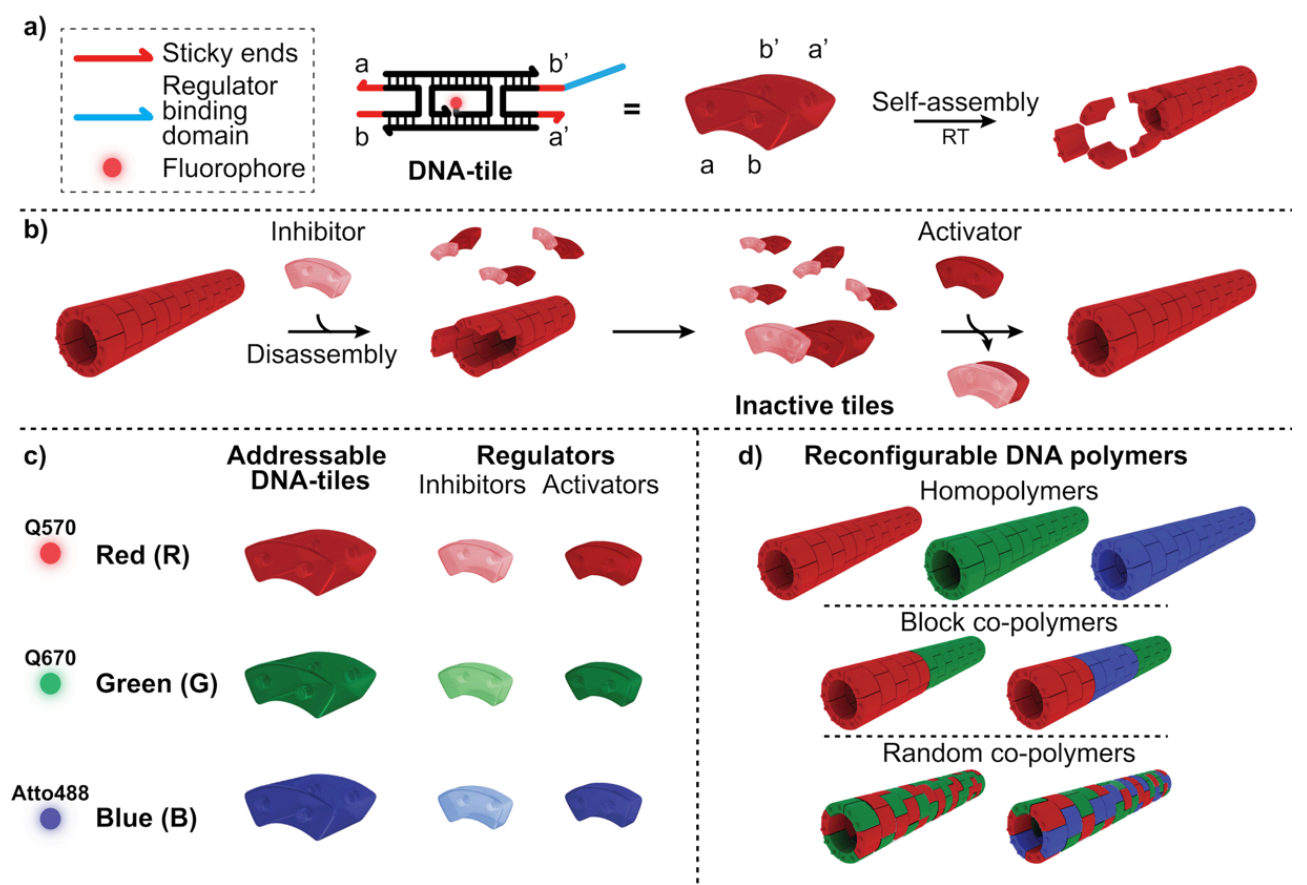

Figure 1. Orthogonally addressable DNA tiles for reorganization of DNA-based polymers. (a) Re-engineered DNA tiles assembled through the interaction of five different strands containing four sticky ends ( $\left.a, b, a^{\prime}, b^{\prime}\right)$ each of 5 nucleotides and a regulator binding domain (see Supporting Information for more details on the sequences). ${ }^{52,54,55}$ The tiles are able to self-assemble at room temperature into hollow tubular polymeric structures. We used a LEGO-like model of the DNA tile for better clarity where the two knobs and two holes of each brick represent the four sticky ends of the DNA tile. (b) The 
tiles can be inactivated by an inhibitor DNA strand (invader) that binds the regulatorbinding domain and blocks one of the sticky ends inducing the rapid disassembly of the

tubular structures. ${ }^{54,55}$ A specific activator strand (anti-invader) displaces the inhibitor through a toehold strand displacement reaction (Fig. S2) and thus re-activates the tiles resulting in the re-assembly of the structure. ${ }^{54,55}$ (c) Three different DNA tiles, sharing the same sticky ends responsible for self-assembly, but differing in the sequence of the regulator-binding domain, can be orthogonally addressed by different DNA regulator strands. Each tile is also labelled with a different fluorophore (i.e. Quasar570, Red, R; Quasar670, Green, G; Atto488, Blue, B). (d) Examples of possible polymeric structures that can be reconfigured by the addressable tiles/regulators.

Thanks to the high specificity and predictability of DNA-DNA interactions, it is possible to design in a rational way the DNA tiles so that they can be orthogonally addressed by different regulator strands. To do so, we have designed three different DNA tiles sharing the same sticky ends responsible for self-assembly but differing in the sequence responsible for the binding of the regulator strand (Fig. 1c). Each of the three DNA tiles is also labelled with a different fluorophore (i.e. Quasar570, Red, R; Quasar670, Green, G; Atto488, Blue, B) so that their presence in the self-assembled structures can be easily discriminated using a confocal microscopy (Fig. 1c). We demonstrate here that such orthogonally addressable tiles respond to different regulators and can be used to dynamically and reversibly reconfigure the formed structures into polymers with predefined tile distribution: homopolymers, random co-polymers and di- and tri-block co-polymers (Fig. 1d).

\section{Homo-random DNA polymer reconfiguration using two addressable tiles}

To first demonstrate reorganization of polymer-like DNA structures with orthogonally addressable DNA tiles we have initially employed a set of two of the above described tiles (red, $R$ and green, G). By allowing each tile to self-assemble at room temperature in two separate solutions it is possible to observe, as expected, the formation of micron-scale red and green homopolymers (Fig. S3). Mixing these two solutions gives well separated structures of similar average length $\left.(<\mathrm{L}\rangle_{\operatorname{Red}}=3.5 \pm 0.3, \mu \mathrm{m} ;\langle\mathrm{L}\rangle_{\text {Green }}=3.0 \pm 0.3, \mu \mathrm{m}\right)$ and number $\left(\langle\mathrm{N}\rangle_{\text {Red }}=29 \pm 3 \times 10^{3}\right.$ count $\left./ \mathrm{mm}^{2} ;\langle\mathrm{N}\rangle_{\text {Green }}=44 \pm 4 \times 10^{3} \mathrm{count} / \mathrm{mm}^{2}\right)$. The first reconfiguration starts with the addition of the two inhibitor regulators (red and green inhibitor) specific for both tiles $(0.7 \mu \mathrm{M})$, which leads to the complete disassembly of both structures (Fig. 2a,b). The successive addition of the two activators (red and green activator) $(3 \mu \mathrm{M})$ induces their concomitant re-activation. Because they both share the 
same sticky ends, the resulting polymeric DNA structure displays a random distribution of the two different tiles along the structure evidenced by the superimpositions of the green and red channel (Fig. 2b, final column). The values of the average length and count of the structures obtained from the merged channels (merged, R/G) are within the standard deviation of the values obtained from each separate channel.

a) Inhibitors $(R, G) \quad$ Activators $(R, G)$
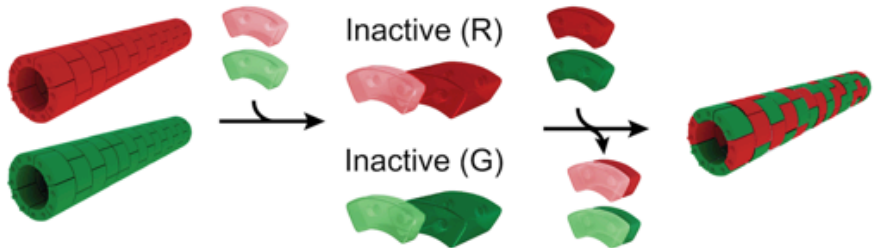

Homo (R/G)

Random (R/G)

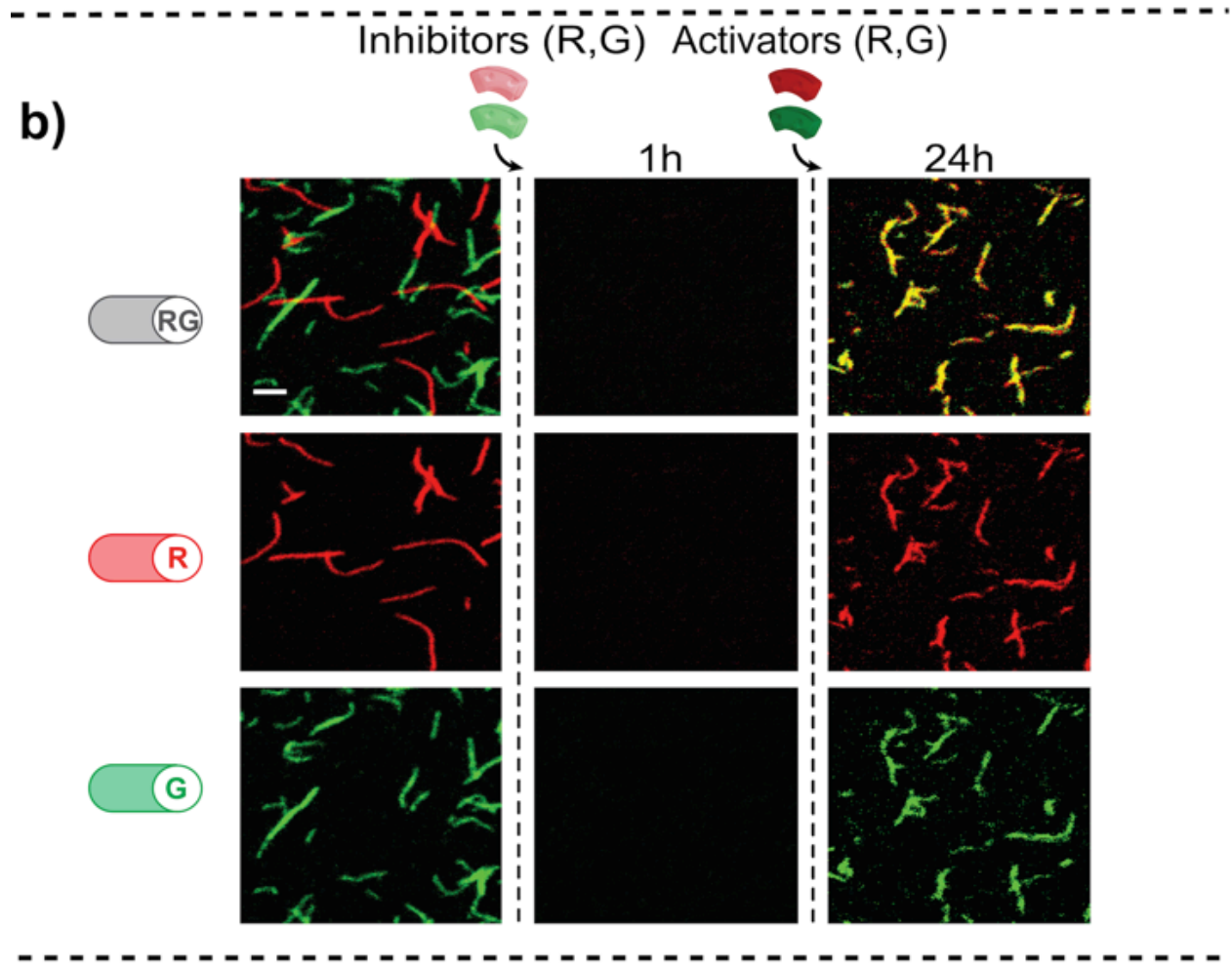

c)

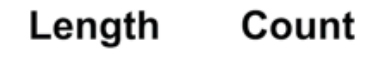

Length
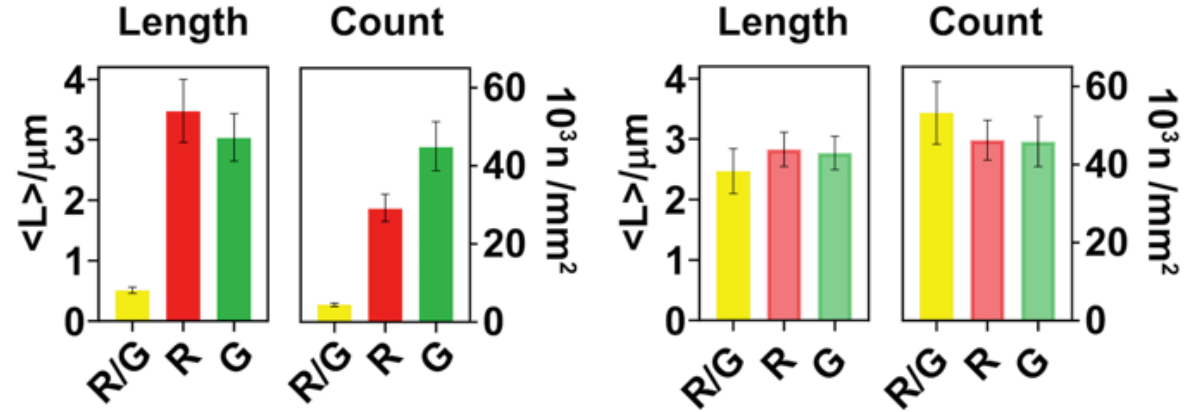

Figure 2. Reorganization from a red and green homopolymers to a random copolymer. (a) Reorganization of two homopolymers (red, $R$ and green, $G$ ) into a statistical random structure can be induced by sequential addition of inhibitors (invader) and activators (anti-invaders) specific for the two addressable tiles. (b) Fluorescence confocal 
images showing the complete disassembly of both structures upon the addition of the two inhibitor $(R, G)$ regulators and the re-assembly into a random structure after the addition of the two activators $(R, G)$. (c) Histograms of the average length ( $\mu m$ ) and count (number of structures $/ \mathrm{mm}^{2}$ ) of the formed DNA structures measured from the corresponding fluorescence microscopy images. $R / G$ bars represent the values obtained by analyzing the structures where co-localization of the red and green tiles occurs. Confocal images were performed in $1 \times \mathrm{TAE}, 12.5 \mathrm{mM} \mathrm{MgCl} 2$ at $\mathrm{pH} 8.0,25^{\circ} \mathrm{C}$ in the presence of an equimolar concentration $(0.25 \mu \mathrm{M})$ of the red and green tiles. The two inhibitors $(0.7 \mu \mathrm{M})$ and the two activators $(3 \mu \mathrm{M})$ were added as indicated in panel a. The error bars indicate the standard deviation of the mean of polymer length and of the count calculated over triplicate experiments. Scale bars, $2.5 \mu \mathrm{m}$.

This supports the random distribution of the two different tiles in the structures (Fig. 2c). To obtain a more quantitative measure of the co-localization we have also calculated the Pearson's coefficient (PC) that estimates the strength of the linear relationship between the fluorescence intensity values of the red and green images. ${ }^{56,57} \mathrm{PC}$ values around 1 would represent high co-localization of the two fluorophores while complete non-colocalization would result in PC values around 0 . As expected, analysis of the two separate homopolymers yields a PC value of $0.09 \pm 0.01$ in support of a very limited colocalization of the two tiles. The random polymers obtained by the sequential addition of the inhibitor and activator strands give, on the contrary, a much higher PC value $(0.75 \pm 0.01)$ indicating that scrambling of the two tiles has occurred.

\section{Homo-random-homo DNA polymer reconfiguration using two addressable tiles}

The reversibility of the reconfiguration of the two addressable DNA tiles can be further demonstrated using more complex reaction schemes. For example, we have mixed in the same solution the red active DNA tiles together with inactive green tiles thus achieving the self-assembly of the red homopolymer coexisting in the same solution with inactive green tiles (Fig. 3a,b). By adding the red inhibitor we can disassemble the red homopolymer leading to a situation where both tiles are inactive (Fig. 3a,b). Subsequent addition of both activators (red and green activators) leads to random hetero polymers with high colocalization ( $P C=0.74 \pm 0.01$ ) (Fig. 3a,b). This random structure can then be reorganized to yield the other homopolymer (i.e. green) by the addition of the red inhibitor (Fig. 3a,b). It is noted that the inactivation of one of the two tiles leads to the complete disassembly of the whole random structure after just 1 minute. This is likely caused by the fact that the two different tiles are randomly distributed in the tubular structure. The disassembled green 
tiles that remain in solution are still in an active conformation and spontaneously reassemble into the green homopolymer coexisting with the red inactive tiles (Fig. 3a,b). In this case we thus demonstrate reconfiguration from one homopolymer (red) to the other (green) through the sequential addition of different orthogonal regulators passing through an intermediate random copolymer (Fig. 3a-c).

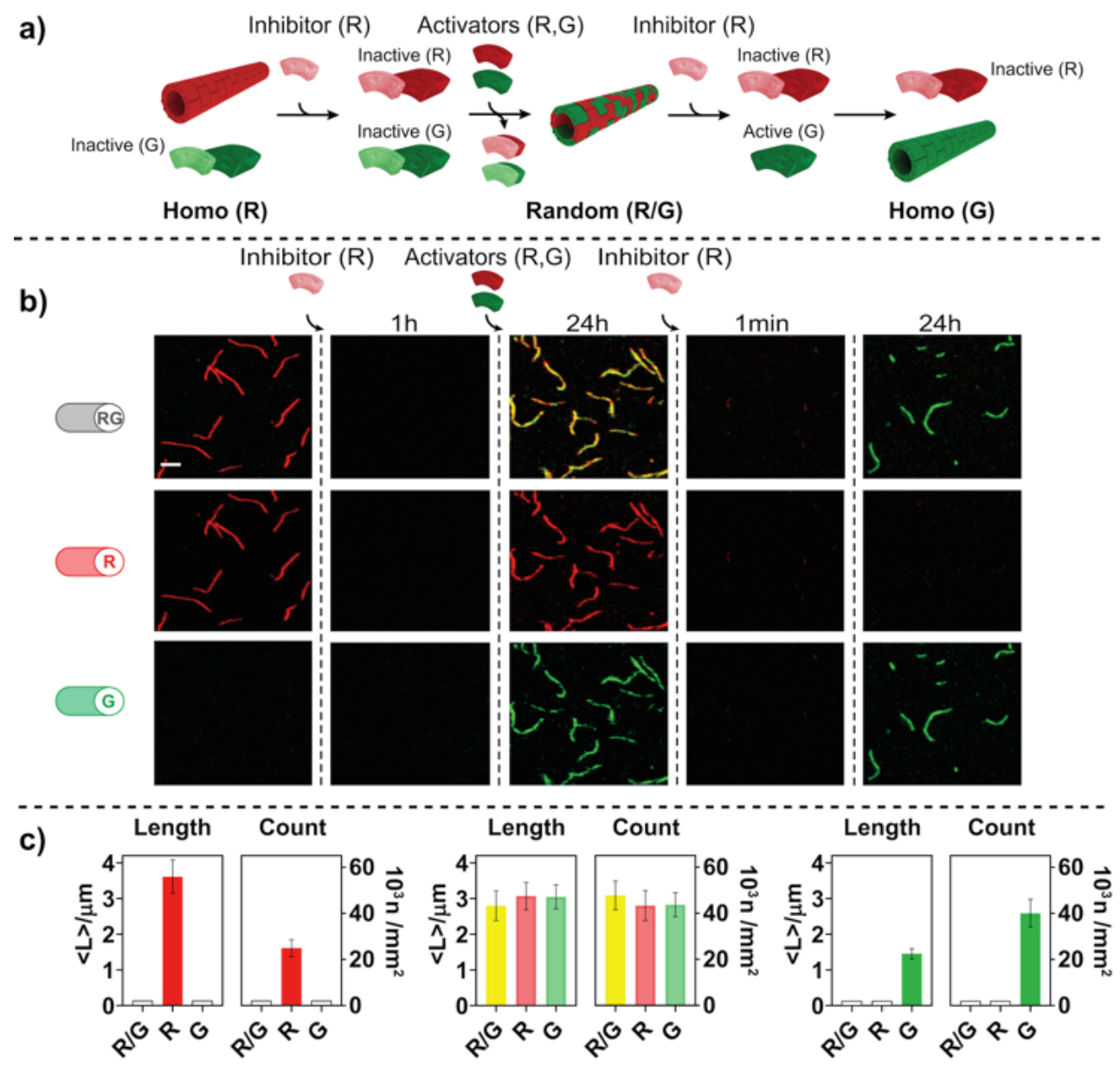

Figure 3. Reorganization from a red homopolymer to a green homopolymer through random co-polymers. (a) Order of sequential addition of the different orthogonal regulators to achieve the described structural reorganization. (b) Fluorescence confocal images showing the disassembly of the red $(R)$ homopolymer upon the addition of the red inhibitor $(R)$, the formation of the $R / G$ random co-polymer obtained after the addition of the red and green activators $(R, G)$ and the self-assembly of the green $(G)$ homopolymer following addition of the red inhibitor (R). (c) Histograms of the average length and count of the formed DNA structures measured from fluorescence microscopy images taken after each step at the indicated interval. R/G bars represent the values obtained by analyzing the structures where co-localization of the red and green tiles occurs. Confocal images were obtained in $1 \times \mathrm{TAE}, 12.5 \mathrm{mM} \mathrm{MgCl}_{2}$ at $\mathrm{pH} 8.0,25^{\circ} \mathrm{C}$ in the presence of an equimolar concentration $(0.25 \mu \mathrm{M})$ of red and green tiles. The red inhibitor $(0.7 \mu \mathrm{M})$, the two activators $(3 \mu \mathrm{M})$ and the red inhibitor $(2.5 \mu \mathrm{M})$ were added as indicated in panel $a$. The 
error bars indicate the standard deviation of the mean of polymer length and of the count calculated over triplicate experiments. Scale bars, $2.5 \mu \mathrm{m}$.

Alternatively, by sequentially adding the red inhibitor, the two activators (red and green) and the green inhibitor, we achieved reconfiguration from one homopolymer (red) to the same homopolymer (red) passing through a complete disassembly and assembly of the random co-polymer (Fig. S4).

\section{Random-homo-random DNA polymer reconfiguration using two addressable tiles}

To further explore different non-covalent synthesis strategies, we mixed in the same solution the two active DNA tiles (red and green) to obtain, as expected, a random R/G structure with, once again, a high-colocalization of the two different tiles $(P C=0.75 \pm 0.01)$ (Fig. 4a,b) and with similar values of the average length and structure count (Fig. 4c). Also in this case, the addition of only one inhibitor (red) causes the rapid and complete disassembly of the structure and the spontaneous reassembly of the active green tiles over time into a homopolymeric structure (Fig. 4a,b). At this stage, the addition of the green inhibitor and the successive addition of the green and red activator strands leads first to the disassembly of the green homopolymer followed by self-assembly into the original random co-polymer with excellent co-localization (Fig. 4a-c). A similar reorganization of the $R / G$ random copolymer passing through the red homopolymer as intermediate can also be obtained by inverting the order of the two inhibitors used (Fig. S5). 


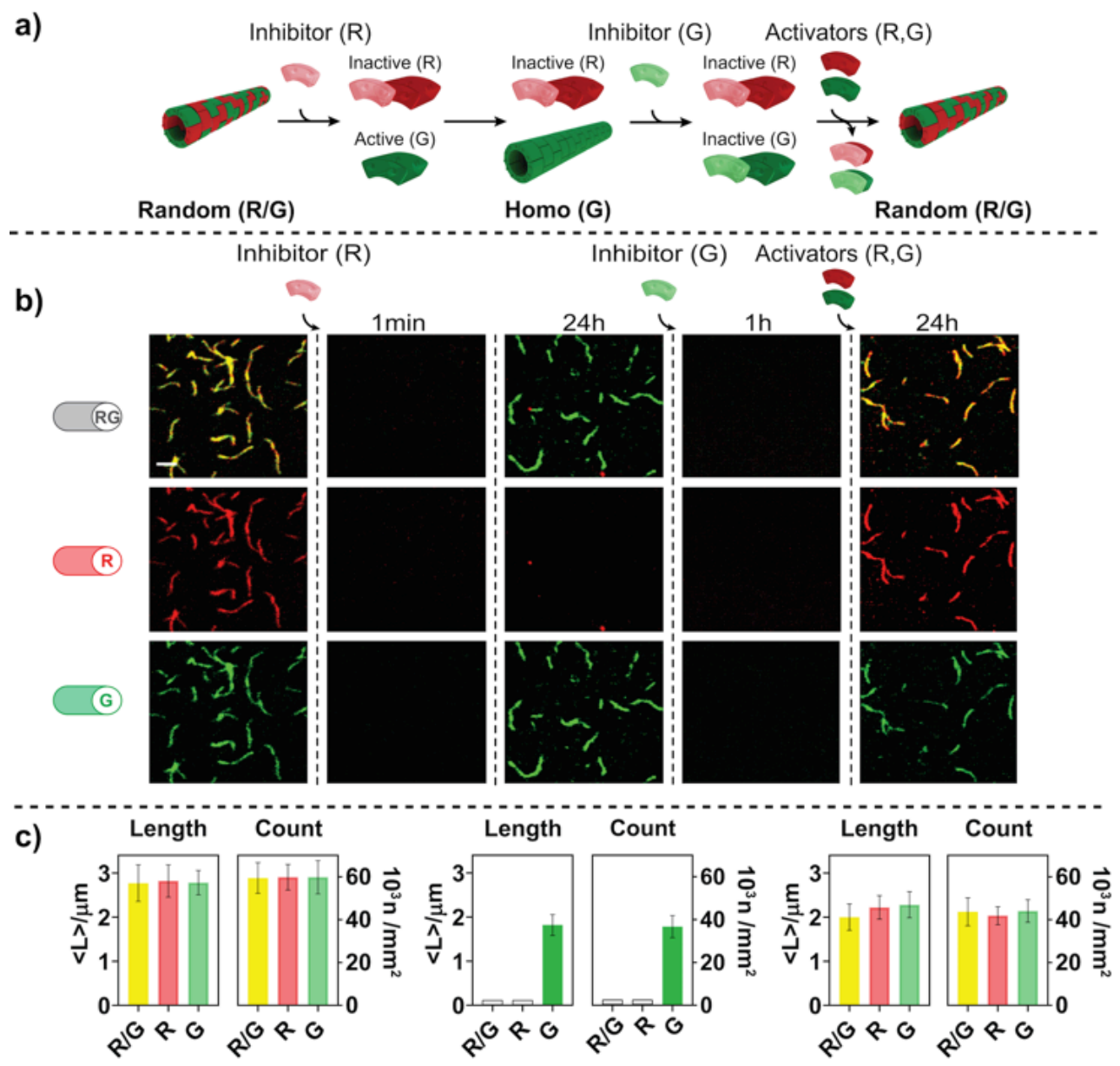

Figure 4. Reversible cyclic reorganization from/to a random co-polymer passing through a homopolymer. (a) Order of sequential addition of the different orthogonal regulators to achieve the described structural reorganization. (b) Fluorescence confocal images showing the disassembly of the $R / G$ random co-polymer and spontaneous reassembly into the green homopolymer upon the addition of the red inhibitor (R) (center) and the successive disassembly and re-assembly into a random co-polymer after the sequential addition of the green inhibitor $(G)$ and the red and green activators $(R, G)$. (c) Histograms of the average length and count of the formed DNA structures measured from fluorescence microscopy images taken after each step at the indicated interval. R/G bars represent the values obtained by analyzing the structures where co-localization of the red and green tiles occurs. Confocal images were obtained in $1 \times \mathrm{TAE}, 12.5 \mathrm{mM} \mathrm{MgCl}$ at $\mathrm{pH}$ $8.0,25^{\circ} \mathrm{C}$ in the presence of an equimolar concentration $(0.25 \mu \mathrm{M})$ of red and green tiles. The red inhibitor $(2.5 \mu \mathrm{M})$, the green inhibitor $(1.5 \mu \mathrm{M})$ and the two activators $(4 \mu \mathrm{M})$ were added as indicated in panel a. The error bars indicate the standard deviation of the mean of polymer length and of the count calculated over triplicate experiments. Scale bars, $2.5 \mu \mathrm{m}$. 


\section{Random-homo-block DNA polymer reconfiguration using two addressable tiles}

We then wanted to explore whether it was possible to exploit this strategy for the assembly of co-polymers with a non-random distribution. In a next experiment we demonstrate that it is indeed possible to reconfigure a R/G DNA random co-polymer into a di-block co-polymer in which the two different tiles are distributed in an ordered fashion in the tubular structure (Fig. 5). Starting from the random R/G polymer, we have initially added the red inhibitor strand to disassemble the polymer (image taken after $1 \mathrm{~min}$ ) and permit the formation of the green homopolymer (Fig. 5a-c). The red activator was then added inducing the self-assembly of the red tiles at the two ends of the green homopolymer leading to a well ordered di-block co-polymer (Fig. 5a,b). To further characterize the level of organization in this system, we have calculated the pixel intensity over the length of the initial random polymer and the final di-block co-polymer (Fig. 5d,e). The random polymer shows pixel intensity values in the red and green channel that are indistinguishable from each other over the entire length of the structure. The normalized average pixel intensity (\%) values calculated along the line-profile of the structure are, as expected, consistent with a statistical distribution of the two tiles on the polymer (Fig. 5d). Conversely, the di-block co-polymer shows well separated regions in which the structure is predominantly composed of only one tile (Fig. 5e), although we note that, probably due to an incomplete inactivation of the red tiles, a portion of the green segment contains also a small fraction of red tiles. 

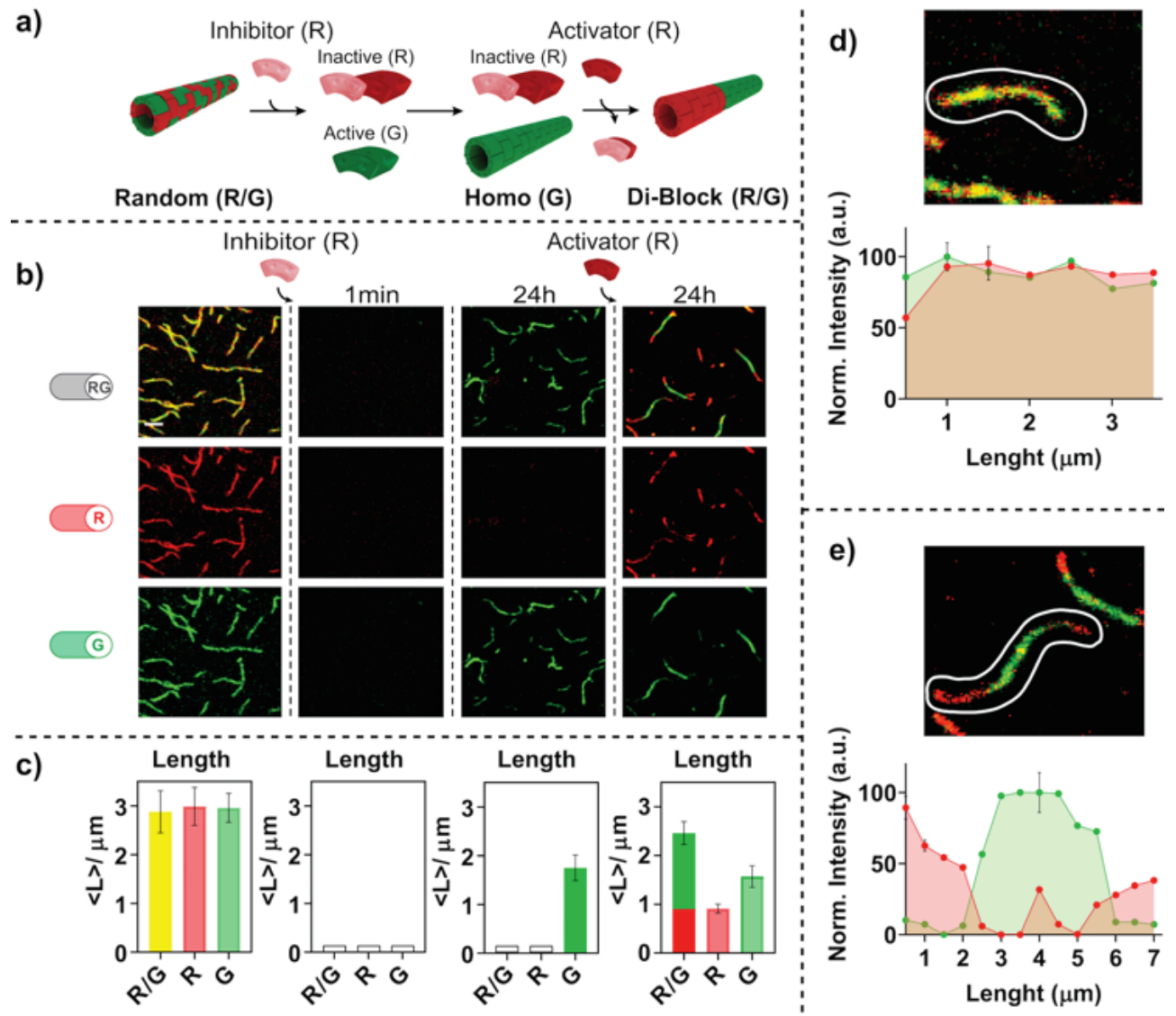

Figure 5. Reorganization from a random co-polymer to a di-block co-polymer. (a) Order of sequential addition of different orthogonal regulators to achieve the described structural reorganization. (b) Fluorescence confocal images showing the disassembly of the $R / G$ random co-polymer and spontaneous re-assembly into the green homopolymer upon the addition of the red inhibitor (R) (center) and the successive self-assembly of the di-block co-polymer upon the addition of the red activator $(R)$. (c) Histograms of the average length and count of the formed DNA structures measured from fluorescence microscopy images taken after each step at the indicated interval. $R / G$ bars represent the values obtained by analyzing the structures where co-localization of the red and green tiles occurs. (d) Average normalized pixel intensity values (\%) of the green and red channels calculated over $0.5 \mu \mathrm{m}$ segments along the line-profile of a $R G$ random polymer (circled in the upper image). (e) Average normalized pixel intensity values (\%) of the green and red channels calculated over $0.5 \mu \mathrm{m}$ segments along the line-profile of a di-block copolymer (circled in the upper image). For a matter of clarity, the error bars in the average normalized pixel intensity values (panel $d, e$ ) have been depicted for only one point on each channel (red and green) of the profile and represent the maximum value of standard deviation. Confocal images were obtained in $1 \times \mathrm{TAE}, 12.5 \mathrm{mM} \mathrm{MgCl} 2$ at $\mathrm{pH} 8.0,25{ }^{\circ} \mathrm{C}$ in the presence of an equimolar concentration $(0.25 \mu \mathrm{M})$ of red and green tiles. The red inhibitor $(2.5 \mu \mathrm{M})$ and the activator $(4 \mu \mathrm{M})$ were added as indicated in panel a. The error bars indicate the standard deviation of the mean of polymer length calculated over triplicate experiments. Scale bars in panel b, $2.5 \mu \mathrm{m}$. 


\section{Random-homo DNA polymer reconfiguration using three addressable tiles}

The high specificity of DNA-DNA interactions allows the design of additional DNA tiles that can be orthogonally addressed with different regulator strands. To demonstrate this, we have simultaneously activated in the same solution three orthogonal DNA tiles (red, green, blue) and have initially demonstrated that they can self-assemble into a random DNA polymer with a statistical distribution of tiles. (Fig. 6a-c). By adding the red inhibitor, we can rapidly disassemble these polymers (image taken after $1 \mathrm{~min}$ ) and observe reassembly of a random polymer formed by the two remaining active tiles (green, blue) (Fig. 6a-c). Further addition of the inhibitor specific for the blue tile induces the disassembly of this random G/B polymer and the subsequent reassembly of the green homopolymer (Fig. $6 a-c)$. In this case we thus achieve reconfiguration from a random polymer made of three tiles $(R / G / B)$ to one random polymer made of two tiles $(G / B)$ and finally to a single homopolymer ( $G$ ) by the sequential deactivation of specific tiles (sequentially $R$ and $B$ ). Of note, also in this case the reconfiguration is achieved passing through a complete disassembly of the polymer after each regulator addition. The rapid and near complete disassembly observed upon addition of the red and blue inhibitors can be taken, again, as an additional evidence for the statistical distribution of the original random DNA polymers. 


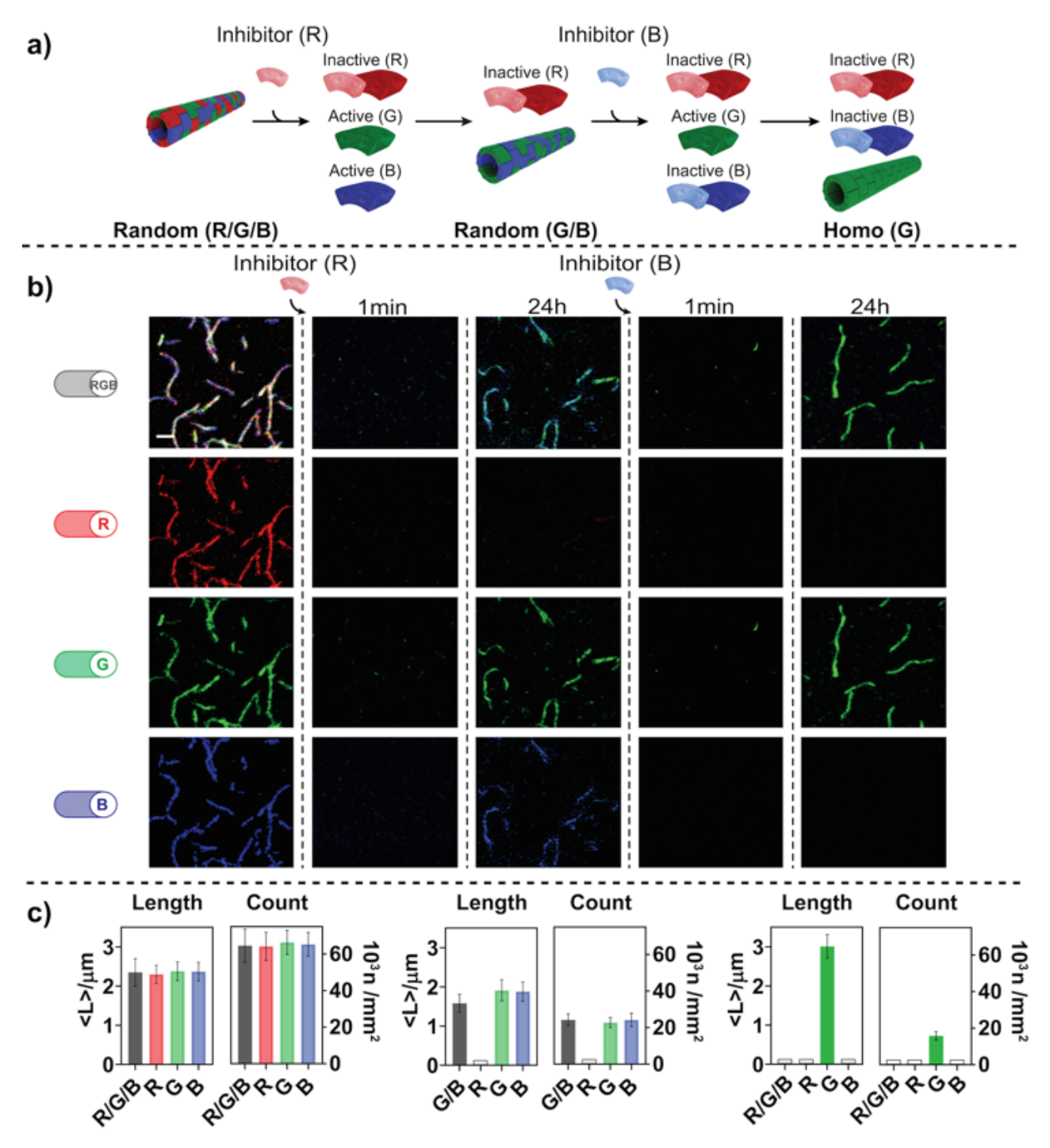

Figure 6. Reorganization from a $R / G / B$ random co-polymer to a $G / B$ random copolymer and to a green homopolymer. (a) Order of sequential addition of the different orthogonal regulators to achieve the described structural reorganization. (b) Fluorescence confocal images showing the disassembly of the $R / G / B$ copolymer and spontaneous reassembly into a random $G / B$ copolymer upon the addition of the red inhibitor $(R)$ (center) and the successive disassembly and re-assembly into the green homopolymer after the addition of the blue inhibitor (B). (c) Histograms of the average length and count of the formed DNA structures measured from fluorescence microscopy images taken after each step at the indicated interval. $R / G / B$ bars represent the values obtained by analyzing the structures where co-localization of the red, green and blue tiles occurs. Confocal images were obtained in $1 \times \mathrm{TAE}, 12.5 \mathrm{mM} \mathrm{MgCl} 2$ at $\mathrm{pH} 8.0,25^{\circ} \mathrm{C}$ in the presence of an equimolar concentration $(0.15 \mu \mathrm{M})$ of the red, green and blue tiles. The red inhibitor $(2.5 \mu \mathrm{M})$ and the blue inhibitor $(3 \mu \mathrm{M})$ were added as indicated in panel a. The error bars indicate the standard deviation of the mean of polymer length and of the count calculated over triplicate experiments. Scale bars, $2.5 \mu \mathrm{m}$. 


\section{Programmable homo-random-homo DNA polymer reconfiguration using three addressable tiles}

The high level of control emerged also from an additional experiment that started with the separate assembly of the three homopolymers (Fig. 7a-c). By sequentially adding the inhibitor and activator strands for the three different tiles it is possible to reorganize them into a random co-polymer in which the three tiles are statistically distributed (Fig. 7a-C). The further addition of the green and blue inhibitors induces the disassembly of the random structure leaving just the red tile activated for self-assembly in the red homopolymer in coexistence with the green and blue inactive tiles (Fig. 7a-c).

a)

Inhibitors (R,G,B) Activator (R,G,B) Inhibitor (G,B)
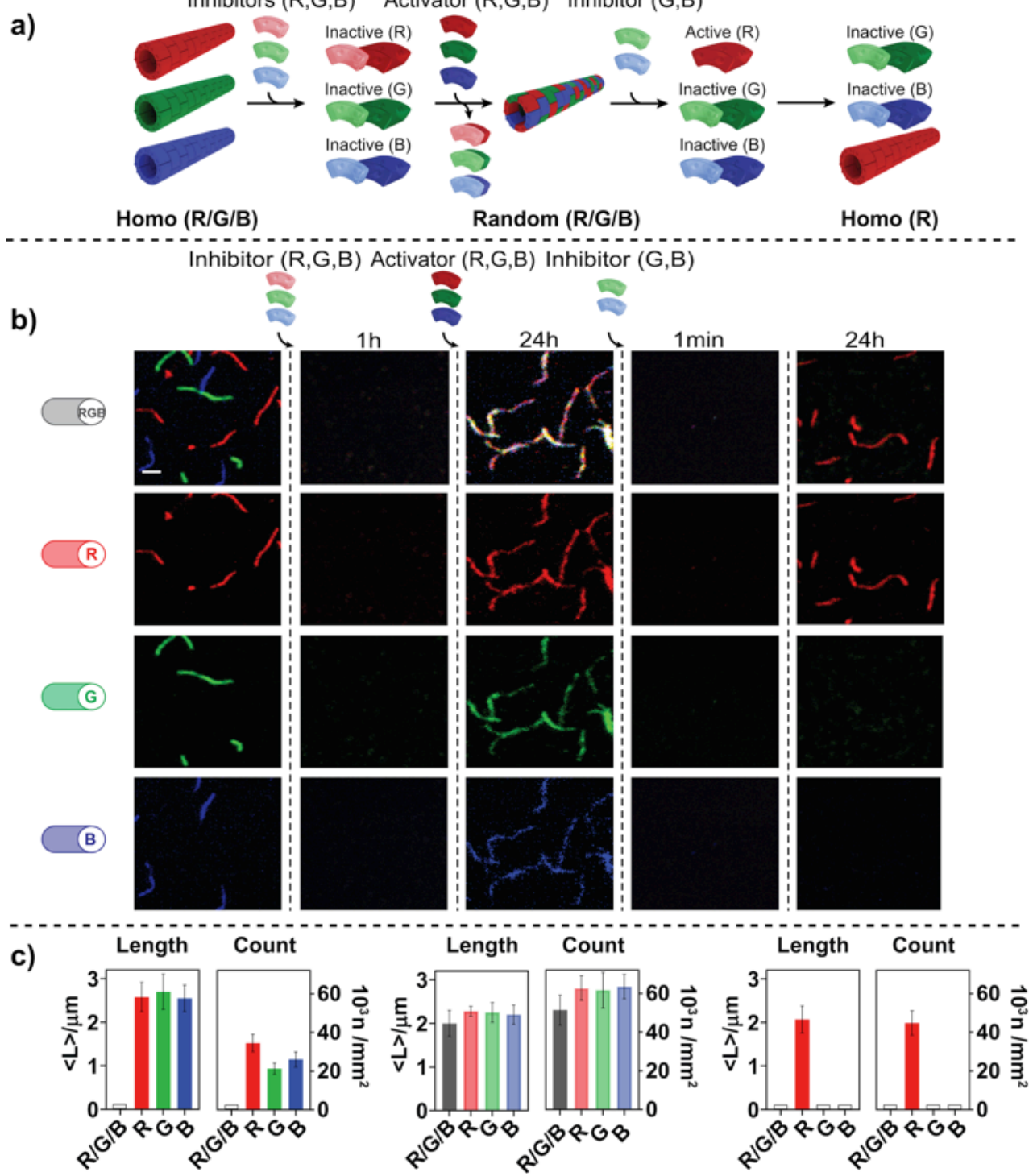

Figure 7. Reorganization from a R/G/B homopolymer to a red homopolymer going through a random $R / G / B$ copolymer. (a) Order of sequential addition of different orthogonal regulators to achieve the described structural reorganization. (b) Fluorescence confocal images showing the disassembly of the red, green and blue homopolymers upon 
the addition of the three inhibitors $(R, G, B)$ and the subsequent formation of, the $R / G / B$ random co-polymer obtained after the addition of the three activators $(R, G, B)$. Addition of the green and blue inhibitors $(G, B)$ induces formation the red homopolymer. (c) Histograms of the average length and count of the formed DNA structures measured from fluorescence microscopy images taken after each step at the indicated interval. $R / G / B$ bars represent the values obtained by analyzing the structures where co-localization of the red, green and blue tiles occurs. Confocal images were obtained in $1 \times$ TAE, $12.5 \mathrm{mM}$ $\mathrm{MgCl}_{2}$ at $\mathrm{pH} 8.0,25^{\circ} \mathrm{C}$ in the presence of an equimolar concentration $(0.15 \mu \mathrm{M})$ of red, green and blue tiles. The three inhibitors $(0.6 \mu \mathrm{M})$, the three activators $(3 \mu \mathrm{M})$ and the green and blue inhibitors (3 $\mu M)$ were added as indicated in panel a. The error bars indicate the standard deviation of the mean of polymer length and of the count calculated over triplicate experiments. Scale bars, $2.5 \mu \mathrm{m}$.

\section{Homo-block DNA polymer reconfiguration using three addressable tiles}

In a final experiment we demonstrate that it is possible to reconfigure a $R / G / B$ random co-polymer into a three-tile block co-polymer in which the three different tiles are orderly distributed in segments (Fig. 8). To achieve this we have initially mixed the three inactive tiles in the same solution and sequentially added the activator strand for each tile (Fig. 8a). The first activator (blue) leads to the self-assembly of the blue homopolymer coexisting in solution with the inactive green and red tiles (Fig. 8a-c). Upon addition of the green activator, the green tiles start to self-assemble at the two ends of the blue homopolymer leading to a well-ordered di-block G/B co-polymer. Confocal imaging shows that the green and blue tiles are localized in well separated regions (Fig. 8a-c). The subsequent addition of the red activator induces the addition of a new block onto the previously formed copolymer leading to a three-tile block R/G/B co-polymer (Fig. 8a-c). Once again, the pixel analysis reveals a highly ordered distribution of the three different tiles along the length of the polymer (Fig. 8d,e). We note here that the efficiency of such tri-block polymer reconfiguration is not optimal and would require further investigation. For example, we found the majority of $B G$ diblock polymers with only one green segments instead of the two we would expect at both ends of the blue segment. This is probably due to a preference of the tiles to grow over one side of the already formed tubular structure. 


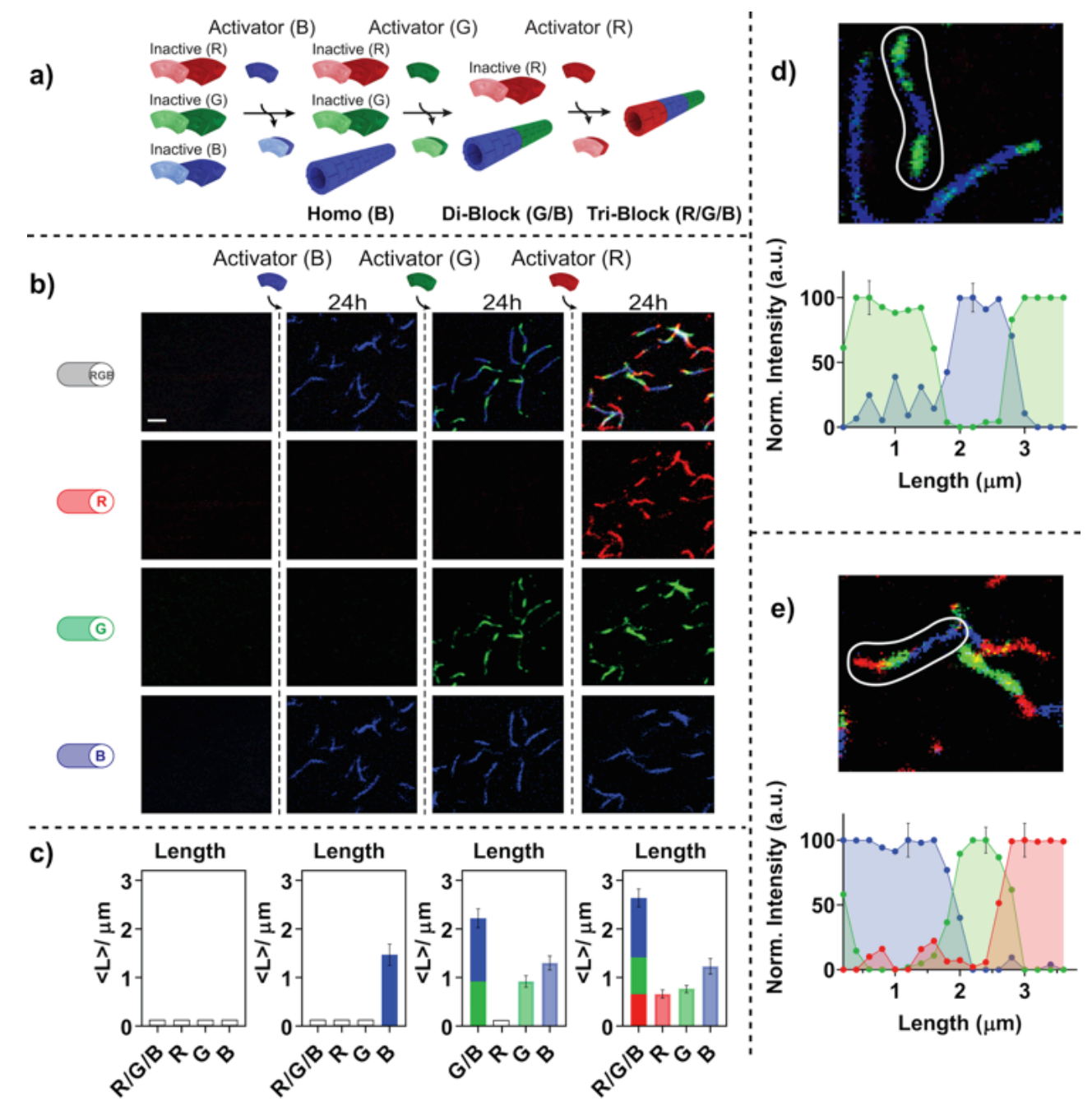

Figure 8. Reorganization from a homopolymer to a three-tile block co-polymer. (a) Order of sequential addition of the different orthogonal regulators to achieve the described structural reorganization. (b) Fluorescence confocal images showing the self-assembly into a blue homopolymer upon the addition of the blue activator $(B)$ and the successive self-assembly of a $G / B$ di-block co-polymer upon the addition of the green activator (G). Last column shows the formation of three-tile $R / G / B$ triblock co-polymers upon addition of the red activator $(R)$. (c) Histograms of the average length and count of the formed DNA structures measured from fluorescence microscopy images taken after each step at the indicated interval. $R / G / B$ bars represent the values obtained by analyzing the structures where co-localization of the red, green and blue tiles occurs. (d) Average normalized pixel intensity values (\%) of the green and blue channels calculated over $0.2 \mu \mathrm{m}$ segments along the line-profile of a di-block co-polymer (circled in the upper image). For a matter of clarity, the error bars in the average normalized pixel intensity values (panel $d, e)$ have been depicted for only one point on each channel (red, green and blue) of the profile and represent the maximum value of standard deviation. (e) Average normalized pixel intensity values (\%) of the green, red and blue channels calculated over 0.2 um segments along the line-profile of a tri-block co-polymer (circled in the upper image). Confocal images were obtained in $1 \times \mathrm{TAE}, 12.5 \mathrm{mM} \mathrm{MgCl} 2$ at $\mathrm{pH} 8.0,25^{\circ} \mathrm{C}$ in the presence of an equimolar concentration $(0.15 \mu \mathrm{M})$ of red, green and blue tiles inactivated with the three 
inhibitors (each at $0.6 \mu \mathrm{M})$. The blue, green and red activators were added as indicated in panel a at a concentration of $1.2 \mu \mathrm{M}$ each. The error bars indicate the standard deviation of the mean of polymer length calculated over triplicate experiments. Scale bars, $2.5 \mu \mathrm{m}$

\section{Conclusion}

Here we have reported a strategy to rationally control the reorganization of DNA-based polymers using orthogonally addressable DNA-tiles. The approach we propose here is versatile and allows to control how different tiles can self-assemble into polymeric structures with controlled distribution. The different tiles share the same 5-nt sticky ends responsible for self-assembly but are rationally designed to contain a specific regulator binding domain that does not show any cross-reactivity with the other tiles. The possibility to address (activate or inhibit) the tiles in a selective way makes it straightforward to organize them into supramolecular structures with different distributions: homopolymers, made of a single tile, random polymers in which different tiles are distributed randomly and block structures where the tiles are organized in specific portions of the polymers.

The main advantage that our strategy offers is the possibility to reconfigure DNAbased polymeric structures in a controlled and reversible manner. Although amazing examples of reorganization and redistribution of man-made polymers and structural motifs have been recently demonstrated exploiting the kinetic and thermodynamic pathway complexity of molecular self-assembly, ${ }^{32}$ our approach defines a highly controllable and orthogonal strategy to reconfigure biopolymers using external orthogonal controllers. More precisely our strategy allows to achieve random or block co-polymers starting from homopolymeric structures, but also to dynamically reconfigure the structures from random to block co-polymers. This approach can also be adapted to other DNA-based polymers that could allow a better control of chain length. For example, programmable chain-growth DNA polymerization leading to different DNA nanopatterns was recently demonstrated using different DNA monomers. ${ }^{58}$

The reliability of DNA-based assembly and the predictability of the involved interactions makes the approach extremely versatile and allow to control the reorganization of the DNA structures in a way that would be difficult to achieve with other synthetic approaches. We also note that, while in this work we have limited ourselves to the design of three different sets of tiles and regulators, there are in principle no specific design constraints that would prevent an increase in the number of orthogonal sets that can be used in the same solution. Moreover, synthetic DNA can also be employed as a 
versatile molecular scaffold that can be conjugated with different recognition elements (i.e. aptamers, antigens, small molecules, etc.) and with different functional biomolecules (i.e.

enzymes, antibodies, etc). ${ }^{59-61}$ This offers the possibility to design a wide range of decorated tiles that can respond to different inputs (for example, diagnostic markers) and exploit our strategy to reorganize the tiles into multicomponent synthetic micron-scale DNA structures with control over the distribution and, thus, function of specific biomolecules.

\section{Acknowledgements}

This work was supported by Associazione Italiana per la Ricerca sul Cancro, AIRC (project n. 21965) (FR), by the European Research Council, ERC (Consolidator Grant project $n$. 819160) (FR). We thank Elena Romano, Department of Biology, University of Rome Tor Vergata, for support in the confocal microscopy images.

\section{References}

(1) Nissen, P.; Hansen, J.; Ban, N.; Moore, P. B.; Steitz, T. A. The structural basis of ribosome activity in peptide bond synthesis. Science 2000, 289, 920-930.

(2) Lehn, J-M. Toward self-organization and complex matter. Science 2002, 295, 24002403.

(3) Vantomme, G.; Meijer, E. W. The construction of supramolecular systems. Science 2019, 363, 1396-1397.

(4) Lehn, J-M. Perspectives in chemistry - steps towards complex matter. Anghew. Chem. Int. Ed. 2013, 52, 2836-2850.

(5) Lutz, J-F.; Lehn, J-M.; Meijer, E. W.; Matyjaszewski K. From precision polymers to complex materials and systems. Nat. Rev. Mater. 2016, 1, 16024-16038.

(6) Prins, L. J.; Reinhoudt, D. N.; Timmerman, P. Noncovalent synthesis using hydrogen bonding. Angew. Chem. Int. Ed. 2001, 40, 2382-2426.

(7) Aida, T.; Meijer, E. W.; Stupp, S. I. Functional supramolecular polymers. Science 2012, 335, 813-817.

(8) Webber, M. J.; Appel, E. A.; Meijer, E. W.; Langer, R. Supramolecular biomaterials. Nat. Mater. 2016, 15, 13-26.

(9) Yan, X.; Wang, F.; Zheng, B.; Huang, F. Stimuli-responsive supramolecular polymeric materials. Chem. Soc. Rev. 2012, 41, 6042-6065. 
(10) Amabilino, D. B.; Smith, D. K.; Steed, J. W. Supramolecular materials. Chem. Soc. Rev. 2017, 46, 2404-2420.

(11) Zhang, X.; Wang, C. Supramolecular amphiphiles. Chem. Soc. Rev. 2011, 40, 94-101. (12) De Greef, T. F. A.; Meijer, E. W. Materials science: supramolecular polymers. Nature 2008, 453, 171-173.

(13) Liu, Y.; Lehn, J-M.; Hirsch, A. K. H. Molecular biodynamers: dynamic covalent analogues of biopolymers. Acc. Chem. Res. 2017, 50, 376-386.

(14) De Greef, T. F. A.; Smulders, M. M. J.; Wolffs, M.; Schenning A. P. H. J.; Sijbesma R. P.; Meijer E. W. Supramolecular polymerization. Chem. Rev. 2009, 109, 5687-5754.

(15) Besenius, P. Controlling supramolecular polymerization through multicomponent selfassembly. Polym. Chem. 2017, 55, 34-78.

(16) Otto, S. Dynamic molecular networks: from synthetic receptors to self-replicators. Acc. Chem. Res. 2012, 45, 2200-2210.

(17) Corbett, P. T.; Laclaire, J.; Vial, L.; West, K. R.; Wietor, J-L.; Sanders, J. K. M.; Otto, S. Dynamic combinatorial chemistry. Chem. Rev. 2006, 106, 3652-3711.

(18) Park, T.; Zimmerman, S. C. Interplay of fidelity, binding strength, and structure in supramolecular polymers. J. Am. Chem. Soc. 2006, 128, 14236-14237.

(19) Krische, M. J.; Lehn, J-M. The utilization of persistent $\mathrm{H}$-bonding motifs in the selfassembly of supramolecular architectures. In: Molecular self-assembly organic versus inorganic approaches. Structure and bonding; Fujita, M., Eds.; Springer: Berlin, Heidelberg, 2000; Vol. 96, pp 3-29.

(20) Ustinov, A.; Weissman, H.; Shirman, E.; Pinkas, I.; Zuo, X.; Rybtchinski, B. Supramolecular polymers in aqueous medium: rational design based on directional hydrophobic interactions. J. Am. Chem. Soc. 2011, 133, 16201-16211.

(21) Lee, C. C.; Grenier, C.; Meijer, E. W.; Schenning, A. P. H. J. Preparation and characterization of helical self-assembled nanofibers. Chem. Soc. Rev. 2009, 38, 671-683. (22) Danila, I.; Riobé, F.; Piron, F.; Puigmartí-Luis, J.; Wallis, J. D.; Linares, M.; Ågren, H.; Beljonne, D.; Amabilino, D. B.; Avarvari, N. Hierarchical chiral expression from the nanoto mesoscale in synthetic supramolecular helical fibers of a nonamphiphilic $\mathrm{C}_{3}$-symmetrical T- functional molecule. J. Am. Chem. Soc. 2011, 133, 8344-8353.

(23) Whittell, G. R.; Hager, M. D.; Schubert, U. S.; Manners, I. Functional soft materials from metallopolymers and metallosupramolecular polymers. Nat. Mater. 2011, 10, 176-188. 
(24) Koevoets, R. A.; Versteegen, R. M.; Kooijman, H.; Speck, A. L.; Sijbesma, R. P.; Meijer E. W. Molecular recognition in a thermoplastic elastomer. J. Am. Chem. Soc. 2005, 127, 2999-3003.

(25) Appel, E. A.; Biedermann, F.; Rauwald, U.; Jones, S. T.; Zayed, J. M.; Scherman, O.

A. Supramolecular cross-linked networks via host-guest complexation with cucurbit[8]uril. $J$. Am. Chem. Soc. 2010, 132, 14251-14260.

(26) Tamesue, S.; Takashima, Y.; Yamaguchi, H.; Shinkai, S.; Harada, A. Photoswitchable supramolecular hydrogels formed by cyclodextrins and azobenzene polymers. Angew. Chem. Int. Ed. 2010, 49, 7461-7464.

(27) Kim, I.; Bang, W-Y.; Park, W. H.; Han, E. H.; Lee, E. Photo-crosslinkable elastomeric protein-derived supramolecular peptide hydrogel with controlled therapeutic CO-release. Nanoscale 2019, 11, 17327-17333.

(28) Ge, Z.; Hu, J.; Huang, F., Liu, S. Responsive supramolecular gels constructed by crown ether based molecular recognition. Angew. Chem. Int. Ed. 2009, 48, 1798-1802. (29) Nakahata, M.; Takashima, Y.; Yamaguchi, H.; Harada, A. Redox-responsive selfhealing materials formed from host-guest polymers. Nat. Commun. 2011, 2, 511.

(30) Del Grosso, Prins, L. J.; Ricci, F. Transient DNA-based nanostructures controlled by redox inputs. Angew. Chem. Int. Ed. 2020, 59, 13238-13245.

(31) Williams, R. J.; Smith, A. M.; Collins, R.; Hodson, N.; Das, A. K.; Ulijn, R. V. Enzymeassisted self-assembly under thermodynamic control. Nat. Nanotecnol. 2009, 4, 19-24. (32) a) Sarkar, A.; Sasmal, R.; Empereur-mot, C.; Bochicchio, D.; Kompella, S. V. K.; Sharma, K.; Dhiman, S.; Sundaram, B.; Agasti S. S., Pavan, G. M.; George, S. J. Selfsorted, random, block supramolecular copolymers via sequence controlled, multicomponent self-assembly. J. Am. Chem. Soc. 2020, 142, 7606-7617; b) Sarkar, A.; Behera, T.; Sasmal, R.; Capelli, R.; Empereur-mot, C.; Mahato, J.; Agasti, S.S.; Pavan, G.M.; Chowdhury, A.; George, S. J. Cooperative Supramolecular Block Copolymerization for the Synthesis of Functional Axial Organic Heterostructures J. Am. Chem. Soc. 2020, 142, 11528-11539.

(33) Frisch, H.; Unsleber, J. P.; Lüdeker, D.; Peterlechner, M.; Brunklaus, G.; Waller, M. Besenius, P. pH-Switchable ampholytic supramolecular copolymers. Angew. Chem. Int. Ed. 2013, 52, 10097-10101.

(34) Shigemitsu, H.; Fujisaku, T.; Tanaka, W.; Kubota, R.; Minami, S.; Urayama, K.; Hamachi, I. An adaptive supramolecular hydrogel comprising self-sorting double nanofiber newworks. Nat. Nanotechnol. 2018, 13, 165-172. 
(35) Elacqua, E; Lye, D. S.; Weck, M. Engineering orthogonality in supramolecular polymers: from simple scaffolds to complex materials Acc. Chem. Res. 2014, 47, 24052416.

(36) Hofmeier, H.; Schubert, U. S. Combination of orthogonal supramolecular interactions in polymeric architectures. Chem. Comm. 2005, 21, 2423-2432.

(37) Adelizzi, B.; Aloi, A.; Markvoort, A. J.; Eikelder, H. M. M. T.; Voets, I. K.; Palmans, A. R. A.; Meijer, E. W. Supramolecular block copolymers under thermodynamic control. J. Am. Chem. Soc. 2018, 140, 7168-7175.

(38) Sun, M.; Deng, J.; Walther, A. Polymer transformers: interdigitating reaction networks of fueled monomer species to reconfigure functional polymer states. Angew. Chem. Int. Ed. 2020, 59, 18161-18165.

(39) Seeman, N. C.; Sleiman, H. F. DNA nanotechnology. Nat. Rev. Mater. 2017, 3, 17068. (40) He, Y.; Tian, Y.; Ribbe, A. E.; Mao C. Highly connected two-dimensional crystals of DNA six-point stars. J. Am. Chem. Soc. 2006, 128, 15978-15979.

(41) Ke, Y.; Ong, L. L.; Shih, W. M.; Yin, P. Three-dimensional structures self-assembled from DNA bricks. Science 2012, 338, 1177-1183.

(42) Rothemund, P. W. K. Folding DNA to create nanoscale shapes and patterns. Nature 2006, 440, 297-302.

(43) Ren, J.; Hu, Y.; Lu, C-H.; Guo, W.; Aleman-Garcia, M. A.; Ricci, F.; Willner, I. pHresponsive and switchable triplex-based DNA hydrogels. Chem. Sci. 2015, 6, 4190-4195. (44) Del Grosso, E.; Ponzo, I.; Ragazzon, G.: Prins, L. J.; Ricci, F. Disulfide-linked allosteric modulators for multi-cycle kinetic control of DNA-based nanodevices. Angew. Chem. Int. Ed. 2020, 59, 21058-21063.

(45) Ranallo, S; Sorrentino, D.; Ricci, F. Orthogonal regulation of DNA nanostructure selfassembly and disassembly using antibodies. Nat. Commun. 2019, 10, 5509.

(46) Gerling, T.; Wagenbauer, K. F.; Neuner, A. M.; Dietz, H. Dynamic DNA devices and assemblies formed by shape-complementary, non-base pairing 3D components. Science 2015, 347, 1446-1452.

(47) Song, J.; Li, Z.; Wang, P.; Meyer, T.; Mao, C.; Ke, Y. Reconfiguration of DNA molecular arrays driven by information relay. Science 2017, 357, 6349.

(48) Liu, Y.; Cheng, J.; Fan, S.; Ge, H.; Luo, T.; Tang, L.; Ji, B.; Zhang, C.; Cui, D.; Ke, Y.; Song, J. Modular reconfigurable DNA origami: from two-dimensional to three-dimensional structures. Angew. Chem. Int. Ed. 2020, 59, 23277-23282. 
(49) Dietz, H.; Douglas, S. M.; Shih, W. M. Folding DNA into twisted and curved nanoscale shapes. Science 2009, 325, 725-730.

(50) Schaffter, S. W.; Schneider, J.; Agrawal, D. K.; Pacella, M. S.; Rothchild, E.; Murphy, T.; Schulman, R. Reconfiguring DNA nanotube architectures via selective regulation of terminating structures. ACS Nano 2020, 14, 13451-13462.

(51) a) Sato, Y.; Sakamoto, T.; Takinoue, M. Sequence-based engineering of dynamic functions of micrometer-sized DNA droplets. Sci. Adv. 2020, 6, 3471; b) Lin, Z.; Emamy, H.; Minevich, B.; Xiong, Y.; Xiang, S.; Kumar, S.; Ke, Y.; Gang, O. Engineering Organization of DNA Nano-Chambers through Dimensionally Controlled and MultiSequence Encoded Differentiated Bonds J. Am. Chem. Soc. 2020, 142, 41, 17531-17542 (52) Rothemund, P. W.; Ekani-Nkodo, A.; Papadakis, N.; Kumar, A.; Fygenson, D. K.; Winfree, E. Design and characterization of programmable DNA nanotubes. J. Am. Chem. Soc. 2004, 126, 16344-16352.

(53) Zhang, D. Y.; Hariadi, R. F.; Choi, H. M. T.; Winfree, E. Integrating DNA stranddisplacement circuitry with DNA tile self-assembly. Nat. Commun. 2013, 4, 1965.

(54) Green, L. N.; Subramanian, H. K. K.; Mardanlou, V.; Kim, J.; Hariadi, R. F.; Franco, E. Autonomous dynamic control of DNA nanostructure self-assembly. Nat. Chem. 2019, 11, 510-520.

(55) Green, L. N.; Amodio, A.; Subramanian, H. K. K.; Ricci, F.; Franco, E. pH-driven reversible self-assembly of micron-scale DNA scaffolds. Nano Lett. 2017, 17, 7283-7288. (56) Bolte, S.; Cordelières, F. P. A guided tour into subcellular colocalization analysis in light microscopy. J. Microsc. 2006, 224, 213-232.

(57) Costes, S. V.; Daelemans, D.; Cho, E. H.; Dobbin, Z.; Pavlakis, G.; Lockett, S. Automatic and quantitative measurement of protein-protein colocalization in live cells. Biophys. J. 2004, 86, 3993-4003.

(58) Zhang, H.; Wang, Y.; ... Tian, Z.; Fan, C. Programming chain-growth copolymerization of DNA hairpin tiles for in-vitro hierarchical supramolecular organization. Nat Commun 2019, 10, 1006.

(59) Saccà, B.; Meyer, R.; Erkelenz, M.; Kiko, K.; Arndt, A.; Schroeder, H.; Rabe, K. S.; Niemeyer, C. M. Orthogonal protein decoration of DNA origami. Angew. Chem. Int. Ed. 2020, 49, 9378-9383.

(60) Rosier, B. J. H. M.; Markvoort, A. J.; Gumí Audenis, B.; Roodhuizen, J. A. L.; den Hamer, A.; Brunsveld. L.; de Greef, T. F. A. Proximity-induced caspase- 9 activation on a DNA origami-based synthetic apoptosome. Nat. Catalysis 2020, 3, 295-306. 
(61) Klein, W. P.; Thomsen, R. P. Turner, K. B.; Walper, S. A.; Vranish, J.; Kjems, J.; Ancona, M. G.; Medintz, I. L. Enhanced catalysis from multienzyme cascades assembled on a DNA origami triangle. ACS Nano 2019, 13, 13677-13689. 


\title{
Supporting Information:
}

\author{
EXPERIMENTAL PROCEDURES
}

\section{Chemicals}

Reagent-grade chemicals ( $\mathrm{NaCl}, \mathrm{HCl}, \mathrm{MgCl}_{2}, \mathrm{Na}_{2} \mathrm{HPO}_{4}$, Trizma hydrochloride, Acetic acid, EDTA) were purchased from Sigma-Aldrich (St Louis, Missouri, USA) and used without further purifications. All experiments unless otherwise noted were obtained in a Tris Acetate-EDTA (TAE)/Mg ${ }^{2+}$ (TAE) buffer $1 \mathrm{X}, 12.4 \mathrm{mM} \mathrm{MgCl} 2$ at $\mathrm{pH} 8$.

\section{DNA oligonucleotides}

HPLC-purified oligonucleotides were purchased from Biosearch Technologies (Risskov, Denmark), Metabion International AG (Planegg, Germany) and Eurofins Scientific (Luxembourg). The oligonucleotides were dissolved in phosphate buffer $50 \mathrm{mM}(\mathrm{pH}=7.0$ $\pm 0.2)$ at $100 \mu \mathrm{M}$ concentrations and stored at $-20^{\circ} \mathrm{C}$ until use.

\section{DNA-based polymers reconfiguration}

The DNA-based structures were prepared as reported elsewhere. ${ }^{52-53}$ Briefly, we employed the DAE-E tile type which consists of five distinct strands of DNA. Following the strategy reported by Franco and co-workers, we have re-engineered three different DNA tiles containing the same 5-nt sticky ends portion responsible for self-assembly but differing in

the sequence that can be orthogonally addressed by different regulator strands. ${ }^{54,55}$ The formation of individual tiles requires thermal annealing but DNA tubular structures selfassembly proceeds at room temperature. The detailed protocols for each reorganization are reported in DNA-based structures reconfiguration section.

\section{Fluorescence imaging of DNA polymeric structures}

For fluorescence microscopy imaging the central strand of each tile (S3, see sequence in Supporting Information) was labeled at 5' end with a different fluorophore (Quasar570, Quasar670, Atto488). A confocal laser scanning microscope Olympus FV-1000 was used. The emitted photons were collected by a $60 \mathrm{x}$, oil objective. The solutions containing the DNA-based polymers were diluted to achieve a final concentration of each tile of $50 \mathrm{nM}$. A $10 \mu \mathrm{L}$ drop of this diluted solution was then deposited between a clean microscope slide 
and a coverslip and the images at three different wavelengths (Laser 488 Argon $\lambda_{\text {ex }}=488$ $\mathrm{nm}, \lambda_{\text {em }}=520 \mathrm{~nm}$; Laser $543 \mathrm{HeNe} \lambda_{\text {ex }}=543 \mathrm{~nm}, \lambda_{\text {em }}=572 \mathrm{~nm}$; Laser 635 Diodo $\lambda_{\text {ex }}=$ $635 \mathrm{~nm}, \lambda_{e m}=668 \mathrm{~nm}$ ) were taken. DNA structure average length, counts and pixel intensity profiles were quantified by image metrology using the SPIP software (www.imagemet.com). Colocalization analysis was performed by using a plugin for ImageJ software named JACoP (Just Another Co-localization Plugin). ${ }^{56}$ Pixel intensity profiles were obtained by measuring the average normalized values (\%) of the pixel intensities through the defined line segments of the selected polymer for each separate channel.

\section{DNA strands for polymer assembly}

The following sequences are the DNA strands used to form the addressable tiles (S1S5). ${ }^{1,2}$ Nucleotides in italics for strands S2 and S4 denotes the sticky end portions. Strand S3 has been used conjugated to a different fluorophore for each tile at the 5' end. Strand S2 also contains the 7-nt regulator binding domain (in bold) that is different for each addressable tile. ${ }^{3-5}$ The inhibitor strand binds to S2 through a 14-nt portion that first binds to the 7-nt regulator binding domain of $S 2$ and then invades the 5-nt sticky end and 2 additional nucleotides. The inhibitor strand contains an extra 6-nt portion (underlined) that remains available for the binding of the activator strand. The activator first binds to this portion and then invades the duplex formed by the inhibitor and strand S2 displacing the inhibitor from the tile. Sequences and modification schemes for DNA tiles, inhibitor and activator strands are reported below:

\section{Red Tile:}
Name
Sequence
S1
5'-CTC AGT GGA CAG CCG TTC TGG AGC GTT GGA CGA AAC T-3'
S2_R
5'-TGG TAT T GTC TG GTA GAG CAC CAC TGA G AGG TA-3'
S3_Q570
5'-Q570-TCC AGA ACG GCT GTG GCT AAA CAG TAA CCG AAG
CAC CAA CGC T-3'
S4
5'-CAG AC AGT TTC GTG GTC ATC G TACCT-3'
S5
5'-CGA TGA CCT GCT TCG GTT ACT GTT TAG CCT GCT CTA C-3'
Inhibitor (R)
5'-ACC AGA CAA TAC CAA TCC GC-3'
Activator (R)
5'-GCG GAT TGG TAT TGT CTG GT-3' 


\section{Green Tile:}

$\begin{array}{ll}\text { Name } & \text { Sequence } \\ \text { S1 } & \text { 5'-CTC AGT GGA CAG CCG TTC TGG AGC GTT GGA CGA AAC T-3' } \\ \text { S2_G } & \text { 5'-CTT ACG T GTC TG GTA GAG CAC CAC TGA G AGG TA-3' } \\ \text { S3_Q670 } & \text { 5'-Q670-TCC AGA ACG GCT GTG GCT AAA CAG TAA CCG AAG } \\ & \text { CAC CAA CGC T-3' } \\ \text { S4 } & \text { 5'-CAG AC AGT TTC GTG GTC ATC G TACCT-3' } \\ \text { S5 } & \text { 5'-CGA TGA CCT GCT TCG GTT ACT GTT TAG CCT GCT CTA C-3' } \\ \text { Inhibitor (G) } & \text { 5'-ACC AGA CAC GTA AGG ATG GC-3' } \\ \text { Activator (G) } & \text { 5'-GCC ATC CTT ACG TGT CTG GT-3' }\end{array}$

Blue Tile:

Name Sequence

S1

S2_B

5'-CTC AGT GGA CAG CCG TTC TGG AGC GTT GGA CGA AAC T-3'

S3_Atto488 5'-Atto488-TCC AGA ACG GCT GTG GCT AAA CAG TAA CCG AAG CAC CAA CGC T-3'

S4 5'-CAG AC AGT TTC GTG GTC ATC G TACCT-3'

S5

Inhibitor (B) 5'-ACC AGA CTT GAA CTT CGA CC-3'

Activator (B) 5'-GGT CGA AGT TCA AGT CTG GT-3'

Protector strands:
Name
Sequence
$\mathrm{P} 1$
5'-TAC CTC TCA GTG GAC AGC CG-3'
P2
5'-GCG TTG GAC GAA ACT GTC TG-3'

\section{DNA-based structures reconfiguration}

For reorganization from red and green homopolymers to a R/G random co-polymer (Figure

2) we have first prepared the two homopolymers in two separate solutions. To do so the 
five strands of each tile were mixed at a concentration of $5 \mu \mathrm{M}$ (tile concentration $=5 \mu \mathrm{M}$ ) in $\mathrm{H}_{2} \mathrm{O} / \mathrm{Mg}^{2+}(12.5 \mathrm{mM} \mathrm{MgCl}$ ) and annealed with a Bio-Rad Mastercycler Gradient thermocycler by heating to $90^{\circ} \mathrm{C}$, and cooling to $20^{\circ} \mathrm{C}$ at a constant rate over a 6-hour period. The so-formed homopolymers were then mixed to a final concentration of each tile of $0.25 \mu \mathrm{M}$ and a microscopy image was taken. The red and green inhibitors (red, R and green, $\mathrm{G}$ ) were then added at a concentration of $0.7 \mu \mathrm{M}$ and the confocal image was taken after 1 hour. Of note, even after 24 hours the tiles do not assemble (data not shown). The red and green activators $(R, G)$ were then added at a concentration of $3 \mu \mathrm{M}$ and the confocal image was taken after 24 hours.

For reorganization from a red homopolymer to a green homopolymer by going through a R/G random co-polymer (Figure 3) the green and red homopolymers were prepared in separate solutions as reported above. The so-formed homopolymers were then mixed to a final concentration of each tile of $0.250 \mu \mathrm{M}$. The green inhibitor $(\mathrm{G})$ was then added at a concentration of $0.7 \mu \mathrm{M}$ and the confocal image was taken after 1 hour. Of note, even after 24 hours the same homopolymer can be observed (data not shown). The red inhibitor (R) was then added at a concentration of $0.7 \mu \mathrm{M}$ and the confocal image was taken after 1 hour. The red and green activators $(R, G)$ were then added at a concentration of $3 \mu \mathrm{M}$ and the confocal image was taken after 24 hours. The red inhibitor $(R)$ was then added at a concentration of $2.5 \mu \mathrm{M}$ and the confocal image was taken after $1 \mathrm{~min}$ and after 24 hours.

For cyclic reorganization from a $R / G$ random co-polymer to the same $R / G$ random copolymer by going through a green homopolymer (Figure 4) we have used the following protocol. The green and red DAE-E tiles were first formed in separate solutions as protected tiles through the interaction between six different DNA strands (four strands of the tile + two protector strands). ${ }^{2}$ The protected tiles are formed by mixing each of the 
above strand (at $5 \mu \mathrm{M}$ ) in $\mathrm{H}_{2} \mathrm{O} / \mathrm{Mg}^{2+}$ (12.5 $\mathrm{mM} \mathrm{MgCl}_{2}$ ) and annealing with a Bio-Rad Mastercycler Gradient thermocycler by heating to $90^{\circ} \mathrm{C}$, and cooling to $20^{\circ} \mathrm{C}$ at a constant rate over a 6-hour period. The green and red protected tiles were then mixed to a final concentration of each tile of $1 \mu \mathrm{M}$. At this solution we have added the deprotector strand (2 $\mu \mathrm{M})$ and allowed the reaction to occur for 24 hours at room temperature. The so-formed $\mathrm{R} / \mathrm{G}$ random co-polymers were then diluted to a final concentration of each tile of $0.25 \mu \mathrm{M}$ and a microscopy image was taken. The red inhibitor $(R)$ was then added at a concentration of $2.5 \mu \mathrm{M}$ and the confocal image was taken after 1 min and 24 hours. The green inhibitor $(G)$ was then added at a concentration of $1.5 \mu \mathrm{M}$ and the confocal image was taken after 1 hour. Of note, even after 24 hours the tiles do not re-assemble (data not shown). The red and green activators $(R, G)$ tiles were then added at a concentration of 4 $\mu \mathrm{M}$ and the confocal image was taken after 24 hours.

For reorganization from a R/G random co-polymer to a di-block co-polymer by going through a green homopolymer (Figure 5) we have used the following protocol. The DAE-E tiles were first formed as protected tiles through the interaction between six different DNA strands (four strands of the tile + two protector strands). ${ }^{2}$ The R/G random co-polymers were first formed as reported above. The so-formed R/G random co-polymers were then diluted to a final concentration of each tile of $0.25 \mu \mathrm{M}$ and a microscopy image was taken. The red inhibitor $(R)$ was then added at a concentration of $2.5 \mu \mathrm{M}$ and the confocal image was taken after $1 \mathrm{~min}$ and 24 hours. The red activator $(R)$ was then added at a concentration of $4 \mu \mathrm{M}$ and the confocal image was taken after 24 hours.

For reorganization from a R/G/B random co-polymer to a green homopolymer going through a G/B random co-polymer (Figure 6) we have used the following protocol. The green, red and blue DAE-E tiles were first formed in separate solutions as protected tiles 
through the interaction between six different DNA strands (four strands of the tile + two protector strands). ${ }^{2}$ The protected tiles are formed by mixing each of the above strand (at $5 \mu \mathrm{M})$ in $\mathrm{H}_{2} \mathrm{O} / \mathrm{Mg}^{2+}\left(12.5 \mathrm{mM} \mathrm{MgCl}_{2}\right.$ ) and annealing with a Bio-Rad Mastercycler Gradient thermocycler by heating to $90^{\circ} \mathrm{C}$, and cooling to $20^{\circ} \mathrm{C}$ at a constant rate over a 6-hour period. The green, blue and red protected tiles were then mixed to a final concentration of each tile of $1 \mu \mathrm{M}$. At this solution we have added the deprotector strand $(10 \mu \mathrm{M})$ and allowed the reaction to occur for 24 hours at room temperature. The so-formed R/G/B random co-polymers were then diluted to a final concentration of each tile of $0.15 \mu \mathrm{M}$ and a microscopy image was taken. The red inhibitor $(R)$ was then added at a concentration of $2.5 \mu \mathrm{M}$ and the confocal image was taken after $1 \mathrm{~min}$ and 24 hours. The blue inhibitor (B) was then added at a concentration of $3 \mu \mathrm{M}$ and the confocal image was taken after $1 \mathrm{~min}$ and 24 hours.

For reorganization from red, green and blue homopolymers to a red homopolymer by going through a R/G/B random co-polymer (Figure 7) the green, red and blue homopolymers were first prepared in separate solutions as reported above. The so-formed homopolymers were then mixed to a final concentration of each tile of $0.15 \mu \mathrm{M}$ and a confocal image was taken. The red, green and blue inhibitors $(R, G, B)$ were then added at a concentration of $0.6 \mu \mathrm{M}$ and the confocal image was taken after 1 hour. Of note, even after 24 hours the same image can be observed at the microscopy (data not shown). The red, green and blue activators $(R, G, B)$ were then added at a concentration of $3 \mu \mathrm{M}$ and the confocal image was taken after 24 hours. The green and blue inhibitors $(G, B)$ were then added at a concentration of $3 \mu \mathrm{M}$ and the confocal image was taken after 1 min and after 24 hours. 
For reorganization from inactive tiles to tri-block co-polymers by going through a blue homopolyer and a G/B di-block co-polymer (Figure 8) we have employed the following protocol. The green, red and blue homopolymers were first prepared in separate solutions as reported above. The so-formed homopolymers were then mixed to a final concentration of each tile of $0.15 \mu \mathrm{M}$. The red, green and blue inhibitors $(R, G, B)$ were then added at a concentration of $0.6 \mu \mathrm{M}$ and the confocal image was taken after 1 hour. Of note, even after 24 hours the same image can be observed at the microscopy (data not shown) The blue activator (B) was then added at a concentration of $1.2 \mu \mathrm{M}$ and the confocal image was taken after 24 hours. The green activator $(G)$ was then added at a concentration of $1.2 \mu \mathrm{M}$ and the confocal image was taken after 24 hours. The red activator $(R)$ was then added at a concentration of $1.2 \mu \mathrm{M}$ and the confocal image was taken after 24 hours. 


\section{Supplementary Figures}

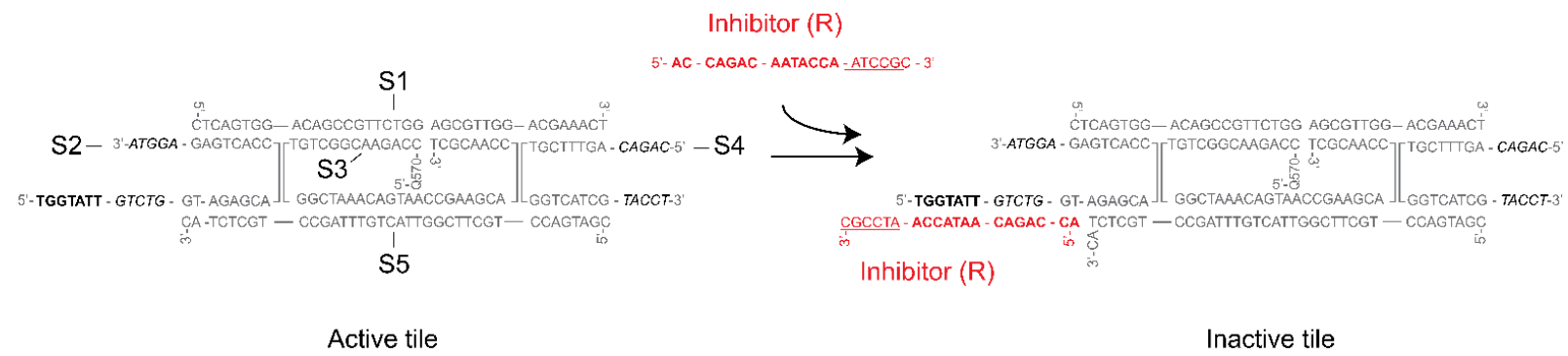

Figure S1. Scheme showing the tile inactivation by the red inhibitor $(R)$ that binds the 7-nt regulator binding domain (bold portion of $S 2$ strand) of the red tile and blocks one of the sticky ends. This leads to either inactive tiles or the disassembly of an already formed polymeric structure. The inactivation mechanism is the same for the green and blue tiles using the green and blue inhibitors, respectively. 


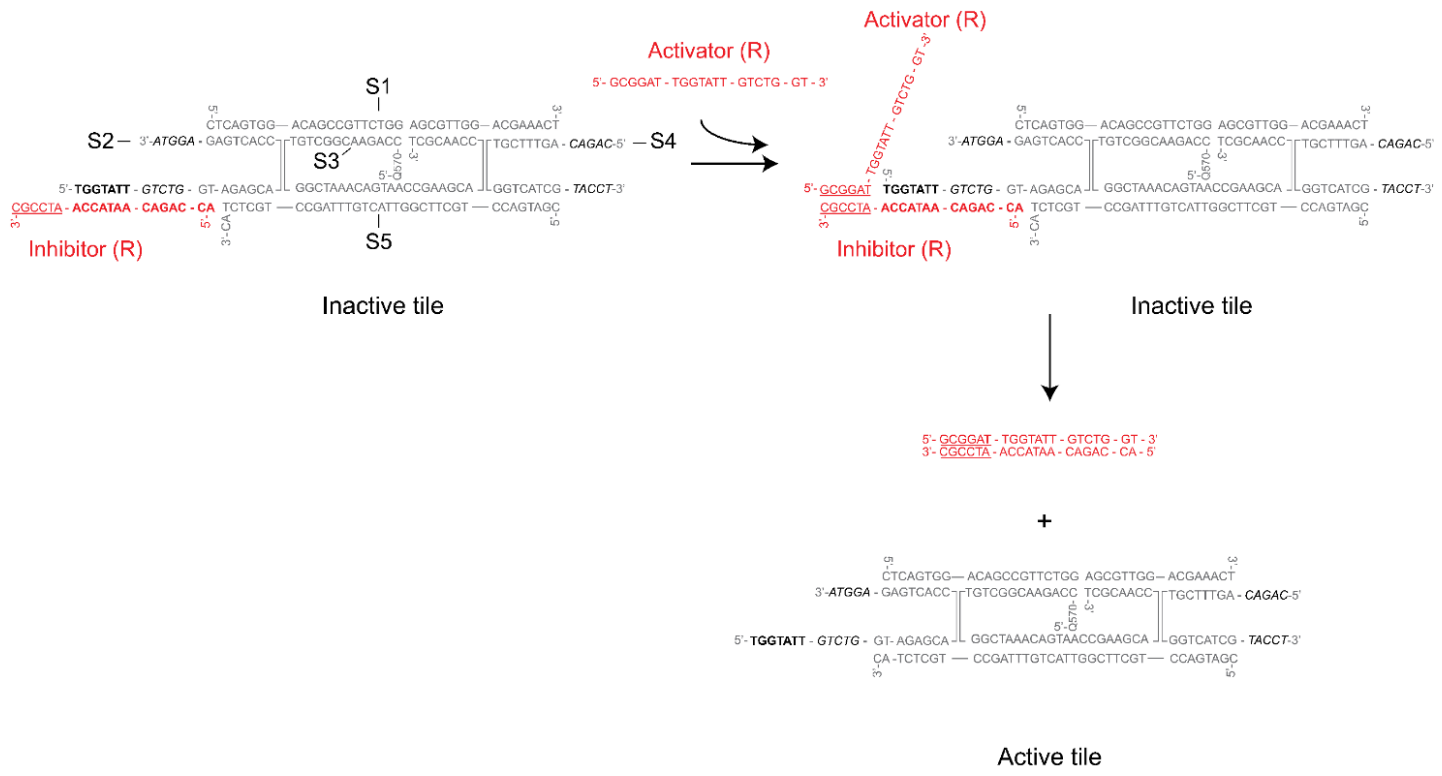

Figure S2. Scheme showing the tile re-activation by the red Activator $(R)$ that binds with a 6-nt portion the free domain of the inhibitor strand (underlined) and displaces the inhibitor from the tile through a toehold-mediated strand displacement reaction. This leads to the activation of the tile and to the re-assembly of the polymeric structure. The re-activation mechanism is the same for the green and blue tiles using the green and blue activators, respectively. 

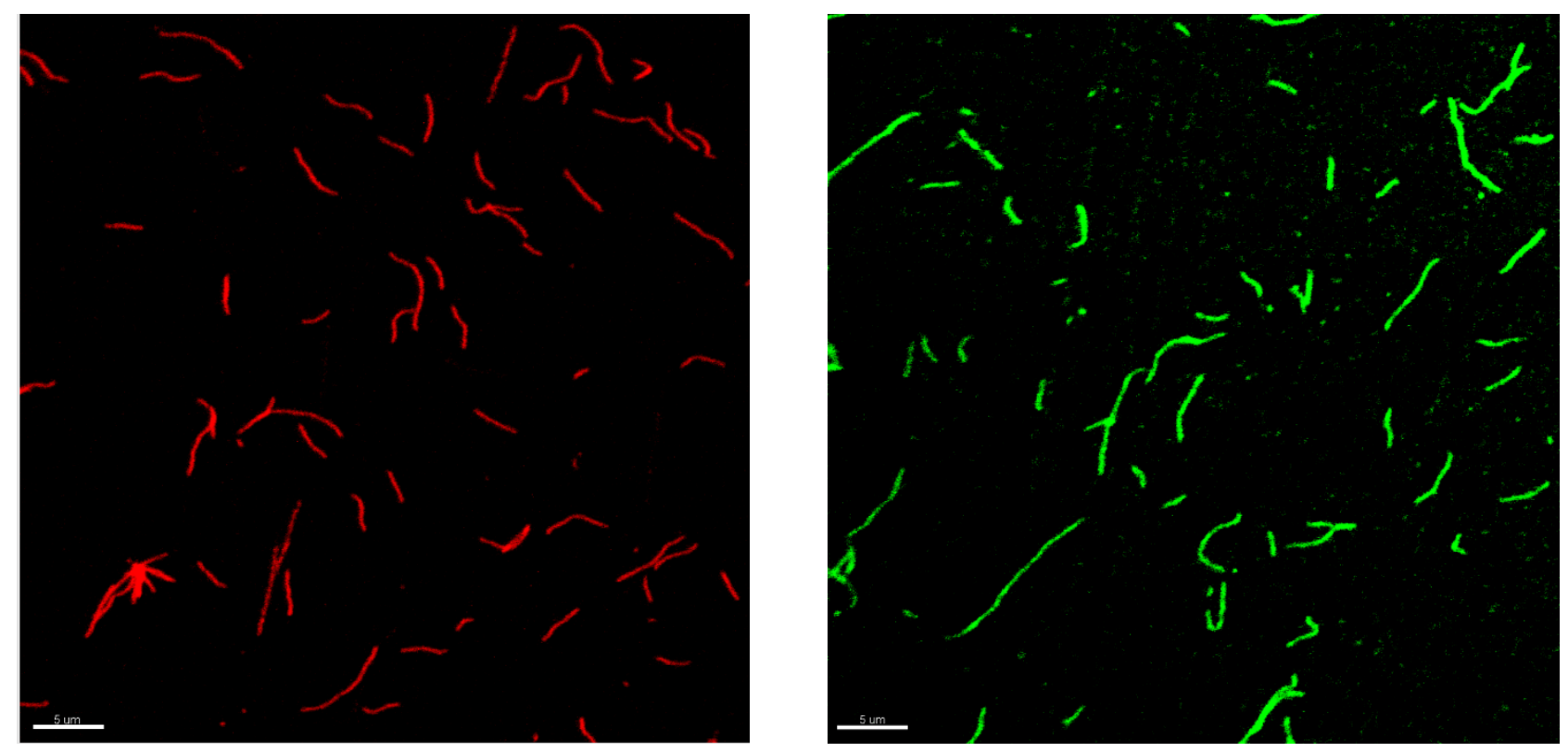

Figure S3. Fluorescence confocal images showing two well-formed red and green homopolymers. The polymeric structures have been prepared in two separate solutions (see Materials and Methods for a description of the procedure) allowing their room temperature self-assembly. Scale bar, $5 \mu \mathrm{m}$. 


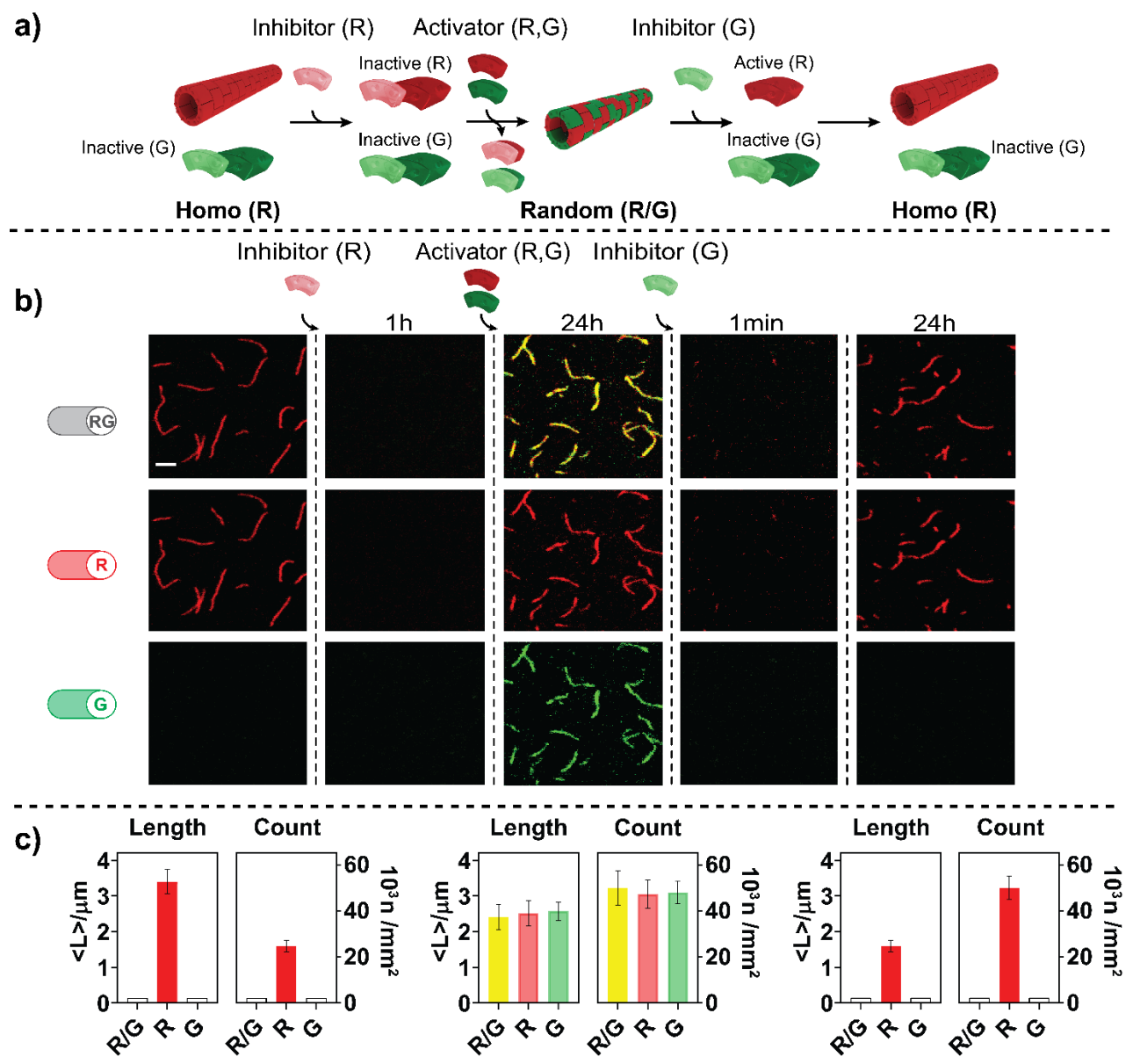

Figure S4. Reversible cyclic reorganization from/to a red homopolymer through random co-polymers. (a) Order of sequential addition of the different orthogonal regulators to achieve the described structural reorganization. (b) Fluorescence confocal images showing the disassembly of the red $(R)$ homopolymer upon the addition of the red inhibitor, the $R / G$ random co-polymer obtained after the addition of the red and green activators and the re-assembly of the red $(R)$ homopolymer following addition of the green inhibitor. (c) Histograms of the average length and count of the formed DNA structures measured from fluorescence microscopy images taken after each step at the indicated interval. $R / G$ bars represent the values obtained by analyzing the structures where colocalization of the red and green tiles occurs. Confocal images were obtained in $1 \times$ TAE, $12.5 \mathrm{mM} \mathrm{MgCl}_{2}$ at $\mathrm{pH} 8.0,25^{\circ} \mathrm{C}$ in the presence of an equimolar concentration $(0.25 \mu \mathrm{M})$ of red and green tiles. The red inhibitor $(0.7 \mu \mathrm{M})$, the two activators $(3 \mu \mathrm{M})$ and the green inhibitor $(3 \mu \mathrm{M})$ were added as indicated in panel a. The error bars indicate the standard deviation of the mean of polymer length and of the count calculated over triplicate experiments. Scale bars, $2.5 \mu \mathrm{m}$. 


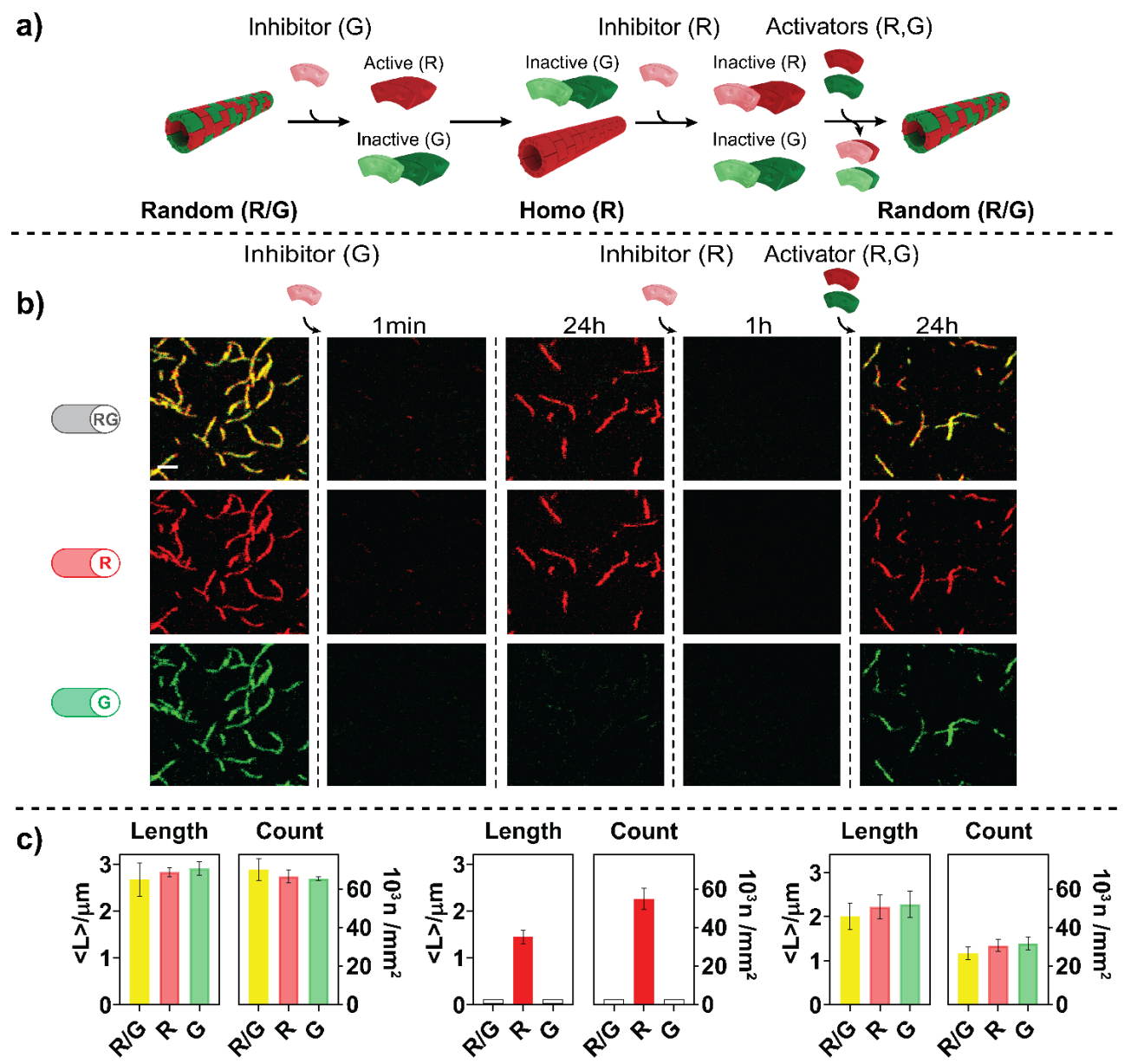

Figure S5. Reversible cyclic reorganization from/to a random co-polymer through a homopolymer. (a) Order of sequential addition of the different orthogonal regulators to achieve the described structural reorganization. (b) Fluorescence confocal images showing the disassembly of the $R / G$ random co-polymer and spontaneous re-assembly into the red homopolymer upon the addition of the green inhibitor (center) and the successive disassembly and re-assembly into a random co-polymer after the sequential addition of the red inhibitor and the red and green activators. (c) Histograms of the average length and count of the formed DNA structures measured from fluorescence microscopy images taken after each step at the indicated interval. $R / G$ bars represent the values obtained by analyzing the structures where co-localization of the red and green tiles occurs. Confocal images were obtained in $1 \times T A E, 12.5 \mathrm{mM} \mathrm{MgCl}_{2}$ at $\mathrm{pH} 8.0,25^{\circ} \mathrm{C}$ in the presence of an equimolar concentration $(0.25 \mu \mathrm{M})$ of red and green tiles. The green inhibitor $(4 \mu \mathrm{M})$, the red inhibitor $(1.5 \mu \mathrm{M})$ and the two activators $(4 \mu \mathrm{M})$ were added as indicated in panel a. The error bars indicate the standard deviation of the mean of polymer length and of the count calculated over triplicate experiments. Scale bars, $2.5 \mu \mathrm{m}$. 


\section{Supplemental References}

(1) Rothemund, P. W.; Ekani-Nkodo, A.; Papadakis, N.; Kumar, A.; Fygenson, D. K.; Winfree, E. Design and characterization of programmable DNA nanotubes. J. Am. Chem. Soc. 2004, 126, 16344-16352.

(2) Zhang, D. Y.; Hariadi, R. F.; Choi, H. M.; Winfree, E. Integrating DNA stranddisplacement circuitry with DNA tile self-assembly. Nat. Commun. 2013, 4, 1965.

(3) Green, L. N.; Amodio, A.; Subramanian, H. K. K.; Ricci, F. \& Franco, E. pH-driven reversible self-assembly of micron-scale DNA scaffolds. Nano Lett. 2017, 17, 7283-7288.

(4) Green, L.N.; Subramanian, H. K. K.; Mardanlou, V.; Kim, J.; Hariadi, R. F.; Franco, E. Autonomous dynamic control of DNA nanostructure self-assembly. Nat. Chem. 2019, 11, 510-520.

(5) Del Grosso, Prins, L. J.; Ricci, F. Transient DNA-based nanostructures controlled by redox inputs. Angew. Chem. Int. Ed. 2020, 59, 13238-13245. 